\title{
Comprehensive profiling of retroviral integration sites using target enrichment methods from historical koala samples without an assembled reference genome
}

Pin Cui, Ulrike Löber, David E Alquezar-Planas, Yasuko Ishida, Alexandre Courtiol, Peter Timms, Rebecca N Johnson, Dorina Lenz, Kristofer M Helgen, Alfred L Roca, Stefanie Hartman, Alex D Greenwood

Background: Retroviral integration into the host germline results in permanent viral colonization of vertebrate genomes. The koala retrovirus (KoRV) is currently invading the germline of the koala (Phascolarctos cinereus) and provides a unique opportunity for studying retroviral endogenization. Previous analysis of KoRV integration patterns in modern koalas demonstrate that they share integration sites primarily if they are related, indicating that the process is currently driven by vertical transmission rather than infection. However, due to methodological challenges, KoRV integrations have not been comprehensively characterized. Results: To overcome these challenges, we applied and compared three target enrichment techniques coupled with next generation sequencing (NGS) and a newly customized sequence-clustering based computational pipeline to determine the integration sites for 10 museum Queensland and New South Wales (NSW) koala samples collected between the 1870 s and late 1980s. A secondary aim of this study sought to identify common integration sites across modern and historical specimens by comparing our dataset to previously published studies. Several million sequences were processed, and the KoRV integration sites in each koala were characterized. Conclusions: Although the three enrichment methods each exhibited bias in integration site retrieval, a combination of two methods, Primer Extension Capture and hybridization capture is recommended for future studies on historical samples. Moreover, identification of integration sites shows that the proportion of integration sites shared between any two koalas is quite small. 
1 Comprehensive profiling of retroviral integration sites using target enrichment

2 methods from historical koala samples without an assembled reference genome

3 Pin Cui $^{1 *}$, Ulrike Löber ${ }^{1,2^{*}}$, David E. Alquezar-Planas ${ }^{1}$, Yasuko Ishida ${ }^{3}$, Alexandre Courtiol ${ }^{4}$,

4 Peter Timms $^{5}$, Rebecca Johnson ${ }^{6}$, Dorina Lenz ${ }^{7}$, Kristofer M. Helgen ${ }^{8}$, Alfred L. Roca ${ }^{3}$, Stefanie

5 Hartmann $^{2}$, and Alex D. Greenwood ${ }^{1}$

$6{ }^{1}$ Department of Wildlife Diseases, Leibniz Institute for Zoo and Wildlife Research (IZW),

7 Berlin, Germany

82 Institute of Biochemistry and Biology, University of Potsdam, Potsdam, Germany

$9{ }^{3}$ Department of Animal Sciences, University of Illinois at Urbana-Champaign, Urbana, Illinois, 10 USA

$11{ }^{4}$ Department of Evolutionary Ecology, Leibniz Institute for Zoo and Wildlife Research (IZW),

12 Berlin, Germany

$13{ }^{5}$ University of the Sunshine Coast, Sippy Downs, Queensland, Australia

$14{ }^{6}$ Australian Museum Research Institute, Australian Museum, Sydney, Australia

$15{ }^{7}$ Department of Evolutionary Genetics, Leibniz Institute for Zoo and Wildlife Research (IZW),

16 Berlin, Germany

$17{ }^{8}$ National Museum of Natural History, Smithsonian Institution, Washington, DC

18 Corresponding Author:

19 Alex D. Greenwood ${ }^{1}$

20 Leibniz Institute for Zoo and Wildlife Research (IZW)

21 in the Forschungsverbund Berlin e.V.

22 Alfred-Kowalke-Straße 17

2310315 Berlin

24 Germany

25 Tel: +49 305168 255; Fax:+49 305126104

26 Email: greenwood@izw-berlin.de

27 *Authors made equal contribution to the study 


\section{ABSTRACT}

Background: Retroviral integration into the host germline results in permanent viral colonization of vertebrate genomes. The koala retrovirus (KoRV) is currently invading the germline of the koala (Phascolarctos cinereus) and provides a unique opportunity for studying retroviral endogenization. Previous analysis of KoRV integration patterns in modern koalas demonstrate that they share integration sites primarily if they are related, indicating that the process is currently driven by vertical transmission rather than infection. However, due to methodological challenges, KoRV integrations have not been comprehensively characterized.

Results: To overcome these challenges, we applied and compared three target enrichment techniques coupled with next generation sequencing (NGS) and a newly customized sequenceclustering based computational pipeline to determine the integration sites for 10 museum Queensland and New South Wales (NSW) koala samples collected between the 1870s and late 1980s. A secondary aim of this study sought to identify common integration sites across modern and historical specimens by comparing our dataset to previously published studies. Several million sequences were processed, and the KoRV integration sites in each koala were characterized.

Conclusions: Although the three enrichment methods each exhibited bias in integration site retrieval, a combination of two methods, Primer Extension Capture and hybridization capture is recommended for future studies on historical samples. Moreover, identification of integration sites shows that the proportion of integration sites shared between any two koalas is quite small.

\section{INTRODUCTION}

Vertebrate endogenous retroviruses (ERVs) descend from exogenous retroviruses that infected the ancestral germ line and have subsequently been transmitted vertically from parent to offspring through Mendelian inheritance (Coffin, Hughes \& Varmus, 1997). ERVs comprise up to $8-11 \%$ of vertebrate genomes (Bromham, 2002; Pontius et al., 2007). Most ERVs colonized their host genomes millions of years ago (Khodosevich, Lebedev \& Sverdlov, 2002; Gifford \& Tristem, 2003) making it difficult to study the process of retroviral invasion. Among vertebrates the exceptions are the EAV-HP virus of chicken and the koala retrovirus (KoRV) which spreads both horizontally and vertically among koalas (Phascolarctos cinereus) (Sacco \& Venugopal, 
57 2001; Tarlinton et al., 2005; Tarlinton, Meers \& Young, 2006; Simmons et al., 2012, Wragg et al. 58 2015), and unlike most other described ERVs, are still in the process of endogenizing into the 59 germ line of the host species (Tarlinton, Meers \& Young, 2008). Therefore, in mammals, KoRV 60 provides a unique opportunity to study the processes underlying ongoing retroviral 61 endogenization. Historical DNA analysis from museum koala samples collected during the $19^{\text {th }}$ and $20^{\text {th }}$ centuries demonstrated that KoRV was already ubiquitous in northern Australia by the $19^{\text {th }}$ century (Ávila-Arcos et al., 2013), and that the KoRV genome has remained strongly conserved (Tsangaras et al., 2014b). In contrast, KoRV integration sites among individuals are highly variable (Tsangaras et al., 2014b; Ishida et al., 2015).

Identical regulatory sequences at the 5' and 3' ends of the proviral genome, designated long terminal repeats (LTRs), are used to mediate viral integration within a host. The distribution of retroviral integration sites in the host genome is generally regarded as non-random (Cereseto \& Giacca, 2004), with several factors influencing integration site selection, including viral integrase (Lewinski et al., 2006) and host chromosomal features (Santoni, Hartley \& Luban, 2010). Retroviruses belonging to the same group tend to exhibit similar integration site preference (Mitchell et al., 2004; Kvaratskhelia et al., 2014). For example, gammaretroviruses in particular have been shown to preferentially integrate into the vicinity of enhancers, gene promoters and CpG Islands (LaFave et al., 2014). Despite these tendencies in integration site preference, the integration of a retrovirus within a precise location in the host genome is still a random event. All individuals in a host population may share older ERV integration sites as they become fixed in the population over time through drift, as is now true for most human endogenous retroviruses (Blikstad et al., 2008). In contrast, if a retrovirus endogenized very recently, the integration site will be rare among all but related individuals such as offspring, as is the case for KoRV among koalas (Tsangaras et al., 2014b; Ishida et al., 2015). The comprehensive identification of ERV integrations within host genomes would allow for research on how ERVs are affected over time by drift, selection and gene flow. Although KoRV integration sites have been examined in koalas, previous studies have not attempted a comprehensive survey of integration sites within or between host individuals. The focus of the current study was to evaluate methods that may comprehensively characterize retroviral integrations and which could be applied to museum samples to examine historical trends in the

87 frequency of shared and unique KoRV integration sites. 
Inverse PCR has conventionally been used for retrieving retroviral integration 89 sites (Nowrouzi et al., 2006). Methods such as rapid amplification of cDNA ends (RACE), 90 ligation-mediated PCR, linker-selection-mediated PCR, linear amplification-mediated PCR and 91 genome walking (Bushman et al., 2005; Moalic et al., 2006; Schmidt et al., 2007; Kustikova, 92 Modlich \& Fehse, 2009; Hüser et al., 2010; Ciuffi \& Barr, 2011) have also been used. However, 93 it is unclear whether these methods can comprehensively detect integration sites given the 94 potential for primer-target mismatch, and they have never been applied to ancient DNA (aDNA). 95 DNA extracted from museum samples has the characteristics of aDNA, e.g. it is heavily 96 fragmented (with most molecules shorter than $300 \mathrm{bp}$ ), damaged (e.g. uracil deamination), and in 97 extremely low concentration (Willerslev \& Cooper, 2005). The DNA degradation, fragmentation 98 and contamination that occurs post mortem makes aDNA research technically 99 challenging (Pääbo et al., 2004; Allentoft et al., 2012), often preventing the use of conventional 100 molecular biological methods such as PCR.

101 To overcome the limitations of working with historical DNA, we applied three target 102 enrichment techniques followed by high-throughput Illumina sequencing. The three techniques, 103 Single Primer Extension (SPEX) (Brotherton et al., 2007), Primer Extension Capture 104 (PEC) (Briggs et al., 2009) and hybridization capture (Maricic, Whitten \& Pääbo, 2010) have been applied successfully to aDNA and could potentially be employed to determine sequences flanking targeted ERVs. Although inherently different, both SPEX and PEC are amplification techniques that specifically target a template strand at a locus of interest. The primer in each case will extend until physically halted or until the end of the template molecule is reached. By contrast, hybridization capture represents a range of varying methodologies used to enrich target sequences by 'capturing' the desired target sequence using hybridization to pre-designed probes.

111 In all three methods, unwanted non-target molecules are washed away, while the enriched 112 template is subsequently re-amplified before high-throughput sequencing. For a detailed 113 overview of the three methods see Figure 1. Ten koala museum samples collected between the 1141870 s and the 1980s were successfully examined. Because no assembled koala genome is 115 currently available, an assembled host-reference-independent computational pipeline was 116 established. The primary aim of this study was to compare the enrichment capabilities of these 117 three methods with respect to establishing the number of KoRV integration sites retrieved from 118 ten museum koalas. We additionally sought to determine the number of integration sites that 
119 were shared across koalas or unique to one koala, and to compare our results to those of

120 published studies on integration sites in historical and modern koalas.

\section{MATERIALS \& METHODS}

\section{Samples and ancient DNA extraction}

123 A total of thirteen museum samples were examined (Table 1). DNA extractions were 124 performed in the ancient DNA (aDNA) laboratory of the Department of Wildife Diseases of the 125 Leibniz Institute for Zoo and Wildlife Research in Berlin, Germany. The laboratory is dedicated 126 to aDNA work and has never been used for molecular work on modern samples. The room is UV 127 irradiated 4 hours every night by ceiling-mounted UV lights. All work performed in the facility 128 follows procedures designed to minimize the possibility of contamination, such as the use of 129 laminar flow hoods and use of protective clothing. The samples used in this study were all 130 derived from museum skin samples and thus no living koalas were sampled at any point during 131 this study.

DNA from approximately $250 \mathrm{mg}$ of koala skin tissue $(7 \mathrm{~mm} \times 7 \mathrm{~mm})$ per museum 133 specimen was obtained using a silica-based extraction kit for aDNA (GENECLEAN Ancient 134 DNA Extraction Kit, MP Biomedicals, USA). The protocol followed the manufacturer's 135 instructions and has been successfully applied to a variety of ancient sample types (Wyatt et al.,

136 2008; Roca et al., 2009). Mock extractions were performed with each set of koala museum 137 specimens as negative controls for extraction. Subsequent to each extraction, the isolated DNA 138 was further purified using a MinElute spin column (Qiagen, Hilden, Germany) as described 139 in (Gilbert et al., 2007) to remove potential inhibitors for the subsequent enzymatic reactions. 140 DNA extracts were not quantified because of the small proportion of endogenous DNA 141 compared to exogenous DNA (contaminants such as bacteria, fungi) in typical aDNA samples.

\section{NGS Library preparation}

143 Illumina sequencing libraries were prepared from the extracts using a previously 144 described protocol (Meyer \& Kircher, 2010) with the following modifications: (A) All SPRI 145 purification steps were substituted with spin column purification (MinElute PCR purification kit, 146 Qiagen). (B) Adapter concentration in the ligation reaction was reduced to $0.2 \mathrm{mM}$ per adapter. 147 (C) The purification after adapter fill-in was substituted by heat inactivation at $80^{\circ} \mathrm{C}$ for $20 \mathrm{~min}$. 148 The libraries were then used directly as a template for subsequent amplification following a two- 
149 step strategy, as previously described (Kircher, Sawyer \& Meyer, 2012). A quality control 150 strategy (Meyer et al., 2008) was also applied, which consisted of qPCR to quantify the product 151 after each step of library amplification. Based on qPCR results, three samples for which DNA 152 quality was too poor for analysis were excluded from further processing.

153 In the first round of amplification, AmpliTaq Gold, a non-proof reading enzyme, and 154 indexing primers (Table S1) were applied, adding a distinct P7 index to each library as described 155 in (Meyer \& Kircher, 2010), 10 indices for the 10 working samples and 3 and 4 negative control

156 indices for PEC and SPEX respectively. Adding distinct indices to each library allows for 157 multiple samples to be sequenced in a single sequencing run. The non-proof reading enzyme 158 allows for amplification to be performed on templates containing deoxyuracils, which are 159 common with aDNA (Der Sarkissian et al., 2015). After removal of $1 \mu \mathrm{L}$ for qPCR as a library 160 quality control, the libraries were used as template in $100 \mu \mathrm{L}$ PCR containing 1x Taq buffer II 161 (Applied Biosystems), 5U AmpliTaq Gold (Applied Biosystems), $250 \mathrm{mM}$ each dNTP and $162100 \mathrm{nM}$ each indexing primer. Cycling conditions followed manufacturer's instructions: The pre163 denaturation step lasted $12 \mathrm{~min}$ at $95^{\circ} \mathrm{C}$, followed by 12 cycles of denaturation at $95^{\circ} \mathrm{C}$ for $20 \mathrm{~s}$, 164 annealing at $60^{\circ} \mathrm{C}$ for $30 \mathrm{~s}$ and elongation at $72^{\circ} \mathrm{C}$ for $40 \mathrm{~s}$, with a final extension step of $72^{\circ} \mathrm{C}$ 165 for 5 min. PCR products were purified using the QIAquick PCR purification kit (Qiagen, Hilden, 166 Germany).

In the second round of amplification, $5 \mu \mathrm{L}$ of the purified PCR product from the first round PCR was used as a template for a second PCR. This involved $50 \mu \mathrm{L}$ reactions containing Herculase II Fusion DNA Polymerase (Agilent Technologies Catalog 600677), which has proof reading activity, and primers IS5 and IS6 (Meyer \& Kircher, 2010) at a final concentration of $400 \mathrm{nM}$ each. Cycling conditions included an activation step of $3 \mathrm{~min}$ at $95^{\circ} \mathrm{C}$, followed by 15 20 cycles of denaturation at $95^{\circ} \mathrm{C}$ for $20 \mathrm{~s}$, annealing at $60^{\circ} \mathrm{C}$ for $25 \mathrm{~s}$ and elongation at $72^{\circ} \mathrm{C}$ for

$17330 \mathrm{~s}$, with a final extension step at $72^{\circ} \mathrm{C}$ for $3 \mathrm{~min}$. The number of cycles used in the PCR for 174 every sample was dependent on the concentration of each of the libraries as determined by the 175 qPCR assay. The PCR amplified libraries were then purified using the QIAquick PCR 176 purification kit. Each library was separately used in subsequent PEC and hybridization capture 177 experiments. 


\section{Bait preparation and integration site enrichment}

179 Three methods were compared for retrieving integration sites: single primer extension

180 (SPEX) primer extension capture (PEC), and hybridization capture. All three have been 181 successfully applied to ancient and historical DNA samples and all are applicable to samples that 182 would not be expected to yield results with conventional methods for integration site analysis.

183 The same set of primers was used in PEC and SPEX experiments (Figure 2, Table S2). Because 184 the two LTRs of a provirus are identical, the primers designed for enriching the 5' integrations 185 will also target the 3' LTR retroviral env gene and the primers designed for targeting the 3' 186 integrations will also extend targeting the retroviral gag leader sequence (Figure 2A). For both 187 the 5' and 3' KoRV LTR, two 20 bp primers were developed which overlap such that the 3' end 188 of the first primer overlapped $8 \mathrm{bp}$ with the 5 ' end of the second primer (Figure 2B primers 5.1 189 and 5.2 and 3.1 and 3.2 respectively). To avoid known LTR polymorphisms among KoRV 190 proviruses, the two primers on each side of the LTR were located $17 \mathrm{bp}$ from the 5' end and $19150 \mathrm{bp}$ from the 3' end of the LTRs in conserved regions (Figure 2B). The baits used for 192 hybridization capture were synthesized to generate $32 \mathrm{bp}$ oligonucleotides that spanned the full 193 length of sequence covered by primers 5.1 and 5.2 (32 bp) on the 5' LTR and primers 3.1 and 3.2 194 (32 bp) on the 3' end.

\section{Primer Extension Capture (PEC)}

196 Indexed libraries were pooled in equimolar ratios for primer extension following a 197 published protocol (Briggs et al., 2009). After each step, $1 \mu \mathrm{L}$ of the product was quantified by 198 qPCR. To minimize the amplification bias, each of the captured products was amplified in 199 triplicate, using $5 \mu \mathrm{L}$ of the captured product as template for each reaction, using the same kit 200 and cycling conditions as described previously under NGS library preparation for second round 201 amplification of Illumina indexed libraries, except that we ran 20 cycles of amplification for all 202 samples. Amplified captured libraries were purified using the QIAquick PCR purification kit 203 (Qiagen, Hilden, Germany) and eluted in $50 \mu \mathrm{L}$ of elution buffer (EB) and used as a template for 204 a second round of PEC.

205 Single Primer Extension (SPEX)

206 The SPEX experiments generally followed a published protocol (Brotherton et al., 2007) 207 using DNA extracts prior to Illumina library construction with three modifications: (1) Illumina 208 sequencing adaptors were attached to the 5' end of the primers used in the first round of partially 
209

210

211

212

213

214

215

216

217

218

219

220

221

222

223

224

225

226

227

228

229

230

231

232

233

234

235

236

237

238 239

nested PCR; (2) MyTaq HS Mix (Bioline, BIO-25045) was used instead of Platinum Taq DNA Polymerase High Fidelity in the first round of a partially nested PCR; (3) only one round of a partially nested PCR amplification was performed. The nested PCR products were then quantified by qPCR and indexed using Illumina indexing primers (Table S3). The indexed PCR products were purified using a QIAquick PCR Purification Kit (Qiagen). The amplicons were quantified by qPCR and subjected to a second round of amplification using the same conditions as the first round. The products were purified again using the QIAquick PCR Purification Kit (Qiagen), quantified by qPCR and pooled at equimolar ratios. All PEC and SPEX products were pooled and measured using High Sensitivity DNA chips on an Agilent 2100 Bioanalyzer, then sequenced at the National High-throughput DNA Sequencing Centre, Copenhagen, Denmark using Illumina MiSeq Reagent Kit v2 (300 cycle).

\section{Hybridization capture}

The amplified libraries were pooled in equimolar ratios, totaling a final amount of $2 \mu \mathrm{g}$. An established protocol was followed (Maricic, Whitten \& Pääbo, 2010) except that synthesized oligonucleotide baits were used instead of PCR products and the EB volume for final elution using Qiagen MinElute column was $20 \mu \mathrm{L}$ instead of $15 \mu \mathrm{L}$. After 2 days of hybridization and subsequent elution steps, $1 \mu \mathrm{L}$ of the final eluate was quantified by qPCR and $5 \mu \mathrm{L}$ (in total $15 \mu \mathrm{L}$ ) was amplified in triplicate using the same kit and cycling conditions as described in the NGS library preparation for second round amplification of Illumina indexed libraries. The pooled PCR products were purified using the QIAquick PCR Purification Kit and was measured using the Tapestation 2200 (Agilent Technologies Catalog G2964AA). Hybridization capture libraries were sequenced at the National High-throughput DNA Sequencing Centre, Copenhagen, Denmark using Illumina MiSeq Reagent Kit v2 (300 cycles).

\section{Preprocessing of sequence data}

Adaptor sequences were removed from sequence reads using cutadapt-1.2.1 (Martin, 2011), and quality trimming was performed using Trimmomatic-0.22 with default settings (Bolger, Lohse \& Usadel, 2014). The paired forward and reverse sequence reads were merged using Flash-1.2.5 where possible (Magoč \& Salzberg, 2011), and both the merged and unmerged reads were used for further analysis. PCR duplicates (clonality in the sequencing data) with $100 \%$ sequence identity were removed using cd-hit-v4.6.1 (Li, Jaroszewski \& Godzik, 2001). 
240 Identification of KoRV integration sites

241 Figure 3 and Table S6 summarize the computational pipeline used for the identification

242 of KoRV integration sites. For its implementation, both existing software and customized perl

243 scripts were used that made use of BioPerl (Stajich et al., 2002). Because the nested primers or

244 bait were designed near the ends of LTR, the primer extension products would include either the

245 first $49 \mathrm{bp}$ of the 5' LTR or the last $82 \mathrm{bp}$ of the 3' LTR, which are designated "LTR ends" in

246 Figure 2A. All sequences with a KoRV flank should contain an LTR end, as a result of the

247 primer extension (Figure 2B). Therefore, KoRV integration sites could be identified as the

248 sequence beyond the KoRV LTR end since all integration sites would be attached to an LTR

249 sequence. However, due to DNA degradation in museum samples, some primer extension

250 products may not have a complete LTR end. Furthermore, minor deletions at the end of the

251 integrated LTRs may be present (Fields, Knipe \& Howley, 1996); for example, a 19 bp deletion

252 was found in a KoRV provirus (Ishida et al., 2015). To get around these potential issues,

253 identification of the LTR ends relied on sequentially selecting sample sequences that contain

254 defined LTR segments; this was done in separate steps for the 5' and 3' flank-containing

255 sequences. The LTR end was divided into two segments, designated A and B (Figure 2B): the B

256 segment corresponds to the last 19 bp of the LTR and is referred to as 5B or 3B in the 5' and 3'

257 LTR ends, respectively. The A segment is the remaining section of the LTR end, which has a

258 length of $30 \mathrm{bp}$ in the $5^{\prime}$ end (5A) and $63 \mathrm{bp}$ in the $3^{\prime}$ end (3A).

259 Initially, sequences containing either of the two A regions in the KoRV LTR end (5A or

$2603 \mathrm{~A}$ in Figure 2B) were identified. For this step, optimal local pairwise sequence alignments

261 (Smith-Waterman, EMBOSS (Rice, Longden \& Bleasby, 2000)) were computed between each

262 sample sequence and the A region in either the 5' or 3' LTR end. Sequences were used for

263 further analysis if they could be aligned to at least $20 \mathrm{bp}$ of the $30 \mathrm{bp} \mathrm{5A}$ segment with at least $90 \%$

264 identity, or if they could be aligned to at least 43 of the $63 \mathrm{bp} 3 \mathrm{~A}$ segment with at least $90 \%$

265 identity (Table 2). Despite the differences in the lengths of the 5' and 3' A segments, this

266 alignment criteria was selected as it resulted in approximately the same sequence identity

267 threshold $(-60 \%)$ for both ends. Sequences not passing these criteria were discarded as artifacts.

268 The LTR ends of all sequences meeting these criteria were trimmed to the distal $19 \mathrm{bp}$ and then

269 used for further analysis. A higher sequence identity threshold was not chosen due to potential

270 DNAse degradation of the molecules or ancient DNA based damage lowering homology. A 20 
271 bp sequence length was the minimum on the 5A segment that allowed for LTR identification, 272 whereas the 3A region was longer allowing for a longer minimum segment.

273

274

275

276

277

278

279

280

281

282

283

284

285

286

287

288

289

290

291

292

293

294

295

296

297

298

299

300
From these sequences, B segments of either 3' or 5' LTR ends were identified (3B or 5B in Figure. 2B). For this step, optimal local sequence alignments were computed between each of the trimmed sequence and the B segment in either the 3' or the 5' LTR end. Only sequences that could be aligned to at least $12 \mathrm{bp}$ of the $19 \mathrm{bp}$ long B segment (3B or 5B) with at least $80 \%$ (Table 2) identity were selected. This criteria was chosen by considering the known polymorphisms in this region of KoRV that originate from the mutagenic properties associated with LTR/host flanking region junctions. The last $19 \mathrm{bp}$ of LTR ends were trimmed from all sequences meeting the selection criteria, leaving LTR free KoRV flanks or KoRV genomic DNA adjacent to the LTR.

All sequences that contained the A region, but for which the $\mathrm{B}$ region was not detected using the pairwise alignment strategy, were then subjected to another test. Specifically, these sequences were used as queries for two separate local database searches using BLAST (Altschul et al., 1990). Such sequences represent LTRs that have suffered deletions at the end, a common occurrence in proviruses. One search was against HiSeq sequencing data of a koala from Queensland, Australia with 100X coverage. The data represent raw Illumina sequences and are not annotated or assembled. After adaptor and quality trimming, 6.469 billion reads from this koala, with a mean length of $78 \mathrm{bp}$, were used for this step. Sequences were considered KoRV integration sites when their non-LTR portion could be aligned with greater than $90 \%$ identity to the koala reads over $60 \%$ length of the sample sequence. A second search was against the Tammar wallaby (Macropus eugenii) genome (GenBank: ABQO000000000.2), which represents the closest related species to koala for which a genome has been assembled (Renfree et al., 2011). Although the wallaby and koala lineages diverged more than 50 Mya (Meredith, Westerman \& Springer, 2009), we expected that some of the koala genomic DNA (flanking KoRV) could be aligned to the homologous wallaby regions. Sequences with at least $70 \%$ identity over $50 \%$ length of the sample sequence to the wallaby genome were therefore considered to be KoRV integration sites. An assumption made is that KoRV does not frequently or preferentially insert into repetitive sites which could cause us to underestimate the total number of integrations. This will only be resolvable once an annotated koala genome becomes available. For the sequences 
301 with a match to the wallaby scaffolds or the koala data, the LTR sequences were trimmed and

302 were then concatenated with the KoRV flanks (obtained in previous steps) for further analysis.

\section{Sorting of sequences representing different integration sites}

304 All sequences with matches to the different segments of the 3' and 5'LTR ends and/or to

305 wallaby scaffolds or koala HiSeq data from each of the enrichment techniques were collected.

306 The sequences matching 3' and 5' LTR ends were kept separate, resulting in a total of six

307 different data sets for further analysis (two data sets each for the PEC, SPEX and hybridization

308 capture). LTR ends were removed from all sequences in these data sets. Before using these

309 sequences to identify shared and unique integration sites, all KoRV flanks were sorted into three

310 categories by length (Table 3): (1) including KoRV flank sequences shorter than 4 bp, the typical

311 length of a KoRV target site duplication. These sequences were valid and in the right extension

312 direction but too short for any biological interpretation, and thus were excluded from further

313 analysis. (2) KoRV flanks with length of 4-14 bp. (3) KoRV flank sequences with length of

$31415 \mathrm{bp}$ or longer. Both KoRV flanks $4-14 \mathrm{bp}$ or with length of $15 \mathrm{bp}$ or greater were used for

315 identifying shared and unique integration sites, but only KoRV flanks of minimum length of

$31615 \mathrm{bp}$ were used for subsequent pairing of 5' and 3' integration sites to one KoRV provirus.

317 Additionally, as per the experimental design (Figure 2A), each of the 5' and 3' primer extension

318 products has two directions of extension, $a$ and $b$. Extension a is towards the KoRV flanks

319 yielding integration sites as expected, while extension $\mathrm{b}$ is towards the KoRV proviral genome

320 yielding unwanted products for this integration site study. These sequences were designated as

321 'internal KoRV reads'. However, despite not representing integration sites, extension b products

322 still represent correctly enriched products from the specific enrichment technique.

323 Combining the KoRV flank types 4-14 bp long and 15 bp or longer, the PEC data had

324392 5' flank sequences and 2,347 3' flank sequences; the SPEX data 6521 5' flank sequences

325 and 9,200 3' flank sequences; and hybridization capture 1,158 5' flank sequences and 28 3' flank

326 sequences. A clustering approach was used to sort all sequences in each of the six data sets into

327 groups of similar sequences; each cluster representing a unique integration site. Sequences that

328 did not share significant similarity with any other sequences in the input file were called

329 singletons. For each of the six data sets, all-against-all BLAST comparisons were run, and the

330 BLAST output was used as input for clustering using TRIBE-MCL (Enright, Van Dongen \& 
331 Ouzounis, 2002), separately for each data set. Different combinations of E-values (all against all

332 BLAST) and inflation values (TRIBE-MCL) were used for this step and the optimal parameter 333 combination for each data set was evaluated. For all combinations of E-values and inflation 334 values, multiple sequence alignments were computed for all clusters using MAFFT $335 \mathrm{v7.127b}$ (Katoh et al., 2002). To assess the quality of the clustering, alignments of the 30 largest 336 clusters of each clustering result were visualized in jalview (Waterhouse et al., 2009) and were 337 verified by eye. An alignment was considered high quality if the total number of mismatches and 338 gaps in every sequence of the alignment was no more than $10 \%$ of the sequence length. If all 30 339 clusters were evaluated to be of high quality, the sequence was further analyzed. The parameter 340 combinations for optimal clustering and related all against all BLAST are listed in Table 4.

341 Singletons and non-singleton clusters containing sequences derived from a single 342 individual koala were considered to represent unique integration sites. Clusters containing 343 sequences shared by more than one koala were considered to represent shared integration sites 344 (Table S4 and S5). A consensus sequence was computed from the alignment of each sequence 345 cluster. Singletons and consensus sequences were then further evaluated first by computing 346 pairwise alignments between these sequences and the gag or env part of KoRV genome (Figure 347 2A) (GenBank: AF151794.2). The sequences that could be aligned to the KoRV genes with at

348 least $90 \%$ identity and of any length were categorized as primer extension or flank capture within 349 the KoRV genome. The LTR sequences at the 5' and 3' ends of the KoRV genome are identical 350 or nearly so and therefore $50 \%$ of the PCR products should extend into the KoRV genome 351 (Figure 2A). Sequences that could not be mapped to KoRV genome were potential KoRV 352 integration sites and were evaluated further. For such sequences, a length filtering was performed 353 with a threshold of $15 \mathrm{bp}$, since this is the minimum length that can be effectively identified by 354 BLAST. The sequences longer than $15 \mathrm{bp}$ were first used as query in BLAST to search against 355 the koala shotgun Hiseq data; they were also mapped to wallaby genome (GenBank: 356 ABQO000000000.2) in Geneious version 6.18 (http://www.geneious.com, (Kearse et al., 2012)).

357 Identified sequences for either one of the two computations were considered to be KoRV 358 integration sites. Sequences shorter than $15 \mathrm{bp}$ are too short for efficient mapping or BLAST; 359 however, because they contained an LTR end, were included in the KoRV specific enrichment 360 statistics (Table 2), although they were not further analyzed. 
361 Pairing of 5' and 3' integration sites to one KoRV provirus

362 Ishida, et al. 2015 (Ishida et al., 2015) identified the length of the retroviral target site

363 duplication (a stretch of host DNA directly adjacent to the retrovirus which is duplicated during

364 retroviral integration) for KoRV to be $4 \mathrm{bp}$. Based on this target site duplication length (Figure 4),

365 all 5' and 3' integration sites were examined for shared four bp target site identity. Only KoRV

366 flanks $15 \mathrm{bp}$ or longer were used for pairing 5' and 3' integration sites. The minimum $26 \mathrm{bp}$

367 (30 bp minus the four bp target site duplication) combined length discriminated true wallaby

368 matches from non-significant blastn results.

369 The paired 5'-3' flanking sequences were 1) mapped against the wallaby genome using

370 the mapping tool in Geneious using default settings, where only the paired 5'-3' integration

371 sequence that could be mapped to the wallaby genome with over $70 \%$ of their total length were

372 scored as positively identified; 2) used as query to search in the HiSeq data of Queensland wild

373 koala using BLAST. Here, only the paired 5' -3' integration sites that could be aligned with over

$37490 \%$ identity with the koala HiSeq reads were considered positive.

375 Statistical analysis of shared integration sites

376 Statistical tests were performed to check if the occurrences of KoRV at sampled

377 integration sites increased as the samples became younger among the 10 museum koala samples.

378 Two logistic regression models were employed: one for 5' integration sites and one for 3'

379 integration sites. Both models had the same structure. The occurrence was considered (binary:

$3801=$ presence, $0=$ absence) as the response variable and time as a continuous fixed effect. Because

381 results were qualitatively similar irrespective of expressing "time" as rank or directly as years,

382 for the sake of simplicity, only the latter was reported. The identity of koalas and of insertion

383 sites were considered as two Gaussian random effects, making this logistic regression a

384 Generalised Mixed effect Model (GLMM). The GLMM was fitted using the function HLfit from

385 the R package spaMM 1.4.1 (Rousset \& Ferdy, 2014), considering a Binomial error structure.

386 The effect of time was tested by performing an asymptotic Likelihood Ratio Test (LRT) using

387 the function anova.HLfit from the same package.

\section{RESULTS}

389 NGS sequencing post enrichment by all three tested methods generated hundreds of 390 thousands to millions of reads. The reads displayed the typical length distribution of aDNA (Fig. 
391 S1). After pre-processing steps, 714,929 sequences from the SPEX approach were available for

392 further analysis, 1,188,365 from PEC, and 11,675,245 from hybridization capture.

393 Single primer extension (SPEX)

394 Using SPEX to target the 5' LTR flanks, 66 integration sites unique to a single koala, and

39515 integration sites shared by at least two koalas were identified across the 10 koala samples, for 396 sample descriptions see Table 1. These integration sites derived from consensus sequences 397 generated from sequence clusters with at least $4 \mathrm{bp}$ of sequence (representing the length of the 398 target site duplication of KoRV) (Ishida et al., 2015) flanking the KoRV LTR (categorized as 399 either KoRV flank sequences 4-14 bp long or 15 bp or longer in Table 3). An additional 15,822 400 sequences were less than $4 \mathrm{bp}$; these could not be further analyzed since their length was shorter 401 than the target site duplication, these are listed as KoRV flanks shorter than 4 bp in Table 3. 402 Additionally, 212 reads were identified as part of the envelope gene of the KoRV genome. This 403 results from the presence of identical primer target sites in the 5' and 3' LTRs (Figure 2A), since 404 KoRV 5' and 3' LTRs are identical or nearly so (Ishida et al., 2015). Thus, approximately 50\% 405 of the sequences are expected to be internal KoRV proviral reads that extend from the LTR into 406 the proviral genome rather than into the host flanking region. For clarity, we term these 407 sequences 'internal KoRV reads'. These sequences that extended into the KoRV genome were 408 categorized separately but included in the total enrichment efficiency evaluation because they 409 still represent correct enrichment of target sequences. SPEX for integration sites next the 3' LTR 410 also identified 182 unique and 28 shared 3' LTR flanks; with 1,527 sequences being too short for 411 further analysis (less than $4 \mathrm{bp}$ of flank sequence) and 223 internal KoRV reads that matched the 412 KoRV genome (Table 3).

\section{Primer extension capture (PEC)}

414 PEC was designed to identify flanking regions 5' of integration sites and detected 126 415 unique and 17 shared integration sites. An additional 496 sequences included less than $4 \mathrm{bp}$ of 416 flank that was too short for further analysis, while 141 internal KoRV reads extended into the 417 KoRV genome. PEC targeting regions downstream of the 3' LTR integration sites identified 538 418 unique and 134 shared integration sites. An additional 1,806 reads were less than $4 \mathrm{bp}$, while 1 419 internal KoRV read was identified that matched KoRV (Table 3). 
420

421

422

423

424

425

426

427

428

429

430

431

432

433

434

435

436

437

438

439

440

441

442

443

444

445

446

447

448

449

\section{Hybridization capture}

Using the 5' LTR region as bait, 862 unique and 25 shared 5' flanking regions were identified by hybridization capture. An additional 191 sequences included less than 4 bp of flank and 151 internal KoRV reads were characterized. Using the 3' LTR region as bait, only 24 unique and no shared integration sites were identified by hybridization capture. The strong bias of this method towards identifying 5 ' integration sites has been previously observed (Tsangaras et al., 2014b). Additionally, 41 sequences included less than 4 bp of flank, while 14 sequences were classified as internal KoRV reads (Table 3).

\section{Summary of computational data processing}

At each step of our bioinformatics pipeline, we recorded for each experiment the number of sequences that met our screening criteria (Figure 3). The mean length, minimum length and maximum length of sequences were also calculated at each step (Table S6). Before any screening criteria were applied, SPEX produced 7,628 million, PEC produced 6,956 million reads, and hybridization capture produced 31,096 million. After screening and PCR duplicate removal of this sequencing data, 9.37\% of the initial sequencing reads were kept for SPEX, 17.08\% for PEC, and $37.55 \%$ for hybridization capture. Clonal sequences i.e. duplicate sequences resulting from PCR bias in amplification were more prevalent for products of SPEX than for products of either PEC or hybridization capture.

Two rounds of bioinformatics LTR end identification was performed (Figure 3). After the first round of LTR end identification, 142,577 (19.94\% of the reads after pre-processing) LTR positive sequences were identified for SPEX, 31,787 (2.67\% of the reads after pre-processing) for PEC, and 5,648 $(0.05 \%$ of the reads after pre-processing) for hybridization capture.

Sequences passing the second round of 5' LTR end selection were 22,542 for SPEX, 1,029 for PEC, and 1,495 for hybridization capture, while the sequences passing the second round of 3' LTR end selection were 10,950 for SPEX, 5,559 for PEC, and 83 for hybridization capture. No KoRV LTR ends were detected in negative controls, extraction or PCR controls lacking template, for any experiment.

\section{Cross-technique comparisons}

The efficiency of target enrichment for each technique was calculated as the total number of identified flank sequences divided by the total number of sequences after removal of clonal 
450 sequences. The total number of target enrichment products included KoRV flanking sequences 451 of any length and internal KoRV reads.

As shown in Table 3, PEC enriched the highest total number of 3' integration sites, 672, 453 whereas hybridization capture enriched the most 5 ' integration sites, 887 . As a percentage of the total sequences retrieved, SPEX achieved the highest target enrichment efficiency (4.68\%). Both

PEC and hybridization capture exhibited lower enrichment percentages $(0.55 \%$ and $0.01 \%$ respectively).

Due to a phenomenon known as CapFlank (Tsangaras et al., 2014a), koala genome sequences near the integration sites may be enriched together with KoRV flanks by concatenation of library molecules on the baits. To estimate the numbers of such target flanks, after PCR clonal sequence removal, all sequences were screened using BLAST against the wallaby, which represents the phylogenetically closest species to the koala with an assembled genome. Hybridization capture exhibited the lowest efficiency of on-target enrichment $(0.01 \%$, Table 3) and highest ratio of CapFlank enrichment (16.41\%), while SPEX achieved the highest efficiency of on-target enrichment (4.68\%) and lowest ratio of CapFlank enrichment (0.23\%).

As illustrated in Figure 5, for the 5' LTR integration sites, hybridization capture yielded the highest total number of integration sites, 887 , and contained $91.36 \%$ of the integration sites identified in the SPEX data set and $86.71 \%$ of the integration sites identified in PEC data set. The 3' LTR integration data followed a different profile with PEC generating the highest total number of integration sites, 692 , containing $85.07 \%$ of the integration sites in the SPEX data set and $91.67 \%$ of the integration sites in the hybridization capture data set. The expected enrichment profile and approximate location of the recovered reads based on each of the three methods performance is shown in Figure 6.

\section{Shared and unique integration sites}

After identical integration sites across the data sets generated by the 3 techniques were combined, 52 shared and 865 unique 5' KoRV host flanks could be identified. Shared integration sites accounted for $5.7 \%$ of the total number identified using 5 ' flanking host sequences, a similar percentage as estimated in a previous study (Tsangaras et al., 2014b). Among the 3' 
478 flanking regions, 146 shared and 570 unique integration sites were identified, with shared sites

479 accounting for $20.4 \%$ of total integration sites identified using 3 ' host genomic sequences.

480 Pairing of 5' and 3' flanking regions to identify individual proviral integration sites

$481 \quad$ KoRV typically produces a $4 \mathrm{bp}$ target site duplication upstream and downstream of its 482 integration site (Ishida et al., 2015). All 4 bp putative 5' target site duplications were compared 483 to all 4 bp putative 3' duplications. In cases where there was an exact match at the $4 \mathrm{bp}$, the two 484 flanking regions were concatenated to simulate the sequence that a virus-free host would have at 485 that locus, assuming that the target site duplications actually were from the same locus. A total of 4861,690 concatenated 5' and 3' host flanking sequences were used to query the koala HiSeq data to 487 identify proviral integration sites (Figure 4). There were 63 matches, indicating that the 5' and 3' 488 flanks actually corresponded to integration sites at the same KoRV proviral locus. Of these 63 489 loci, 40 corresponded to proviral integration sites present in a single koala (Data S1), whereas 23 490 corresponded to a proviral integration site detected in at least two koalas (Data S2).

\section{The comparison of integration sites across different studies}

The KoRV integration sites identified by this study were compared to those reported by

493 Tsangaras et al. 2014 (Tsangaras et al., 2014b) and Ishida et al. 2015 (Ishida et al., 2015) (Table

494 S3). Each study used a different set of koalas, and there was no overlap in koala individuals 495 examined by the three studies. All but one of the koala specimens used by Tsangaras et al. 2014 and compared to our results were museum samples. By contrast, all the koalas examined in Ishida et al. 2015 were from modern samples.

For the 3' integration sites, no sharing of integration sites between the museum samples in this study and museum samples in Tsangaras et al. 2014 was detected. Two integration sites were found to be shared between the two youngest museum samples of the current study and PciSN265 (the only modern koala in Tsangaras et al. 2014). Moreover, one integration site was 502 found shared between a modern koala (Pci-SN248) in Ishida et al. 2015 and Pci-SN265 of Tsangaras et al. 2014. One integration site was also found shared between two museum koalas in the current study and modern koalas in Ishida et al. 2015 (Table S4).

Among 5' integration sites, three were shared between the museum samples in this study and those used in Tsangaras et al. 2014. Two integration sites were found to be shared between

507 the museum samples of this study and Pci-SN265, and two integration sites were found shared 
508 between modern koalas (including Pci-SN248) in Ishida et al. 2015 and Pci-SN265. Additionally,

509 four integration sites were found shared between relatively young museum koalas in this study 510 and modern koalas in Ishida et al. 2015. A 5' integration site (KoRV-5-shared_7) was shared by

5119 koalas, including 4 museum koalas in this study, 4 museum koalas in Tsangaras et al. 2014, 512 and one modern koala (Pci-SN404) in Ishida et al. 2015 (Table S5). Statistical modeling of 513 shared KoRV integration sites among 10 koalas showed an increased sharing of integration sites 514 over time. The details are described in (Article S1).

\section{DISCUSSION}

516 The currently available software for identifying viral integration sites using NGS data

517 require an assembled host genome as a reference, e.g., SLOPE (Duncavage et al., 2011), 518 VirusFinder (Wang, Jia \& Zhao, 2013) and VirusSeq (Chen et al., 2012). For the koala however, 519 no assembled genome is available, only raw sequence reads averaging $98 \mathrm{bp}$ in length. We 520 therefore established a customized computational pipeline that was largely reference521 independent, but it made use of the Illumina HiSeq reads of the koala and assembled scaffolds of 522 the wallaby, the closest relative to the koala with an assembled genome (Renfree et al., 2011).

Given the typically degraded state of DNA in museum specimens, many of the captured 524 or extended molecules in this study either did not extend beyond the LTR or extended only a few 525 bases into the flank. However, such sequences still represent successfully targeted enrichment 526 even if they did not provide extensive integration site information. Primers closer to the ends of 527 the LTRs may have retrieved more and longer integration site data. However, polymorphisms 528 within the ends of the LTRs (Ávila-Arcos et al., 2013) may have led to primer mismatch, 529 reducing the effectiveness of all three methods in identifying integration sites. The distance 530 between the primer target and the end of the 5' LTR was $37 \mathrm{bp}$, whereas for the 3' LTR the 531 distance was $70 \mathrm{bp}$. This may explain why the sequencing following hybridization capture 532 yielded more 5' flanking regions than 3' flanking regions. However, primer position may not be 533 the only factor, since both PEC and SPEX yielded more 3' integration sites overall even though 534 the primers were identically positioned. The LTRs of KoRV are distinct from those of its known 535 closest related viruses, the gibbon ape leukemia virus (GALV). However, we cannot exclude the 536 possibility that additional KoRV like LTRs exist in the koala genome associated with distinct 537 ERVs that may lead to an overestimate of integration sites. However, over a decade of molecular 
538 biological analysis of KoRV like viruses in koalas have not identified such closely related ERVs 539 in any species including koalas.

540 Both techniques that involve extension from a primer (SPEX and PEC) were biased 541 toward the 3' integration sites whereas techniques that did not extend from a primer 542 (hybridization capture and genome-walking) were not. The underlying mechanisms generating 543 this bias are not clear. Several koala samples in the current study overlap with those examined by 544 PCR (around 100 bp amplifications) in Ávila-Arcos et al. 2013 (Ávila-Arcos et al., 2013)(Table 1).

545 Several samples in that study failed to yield PCR products but were successful here, likely 546 because shorter sequences, less than $100 \mathrm{bp}$, are easily retrieved by the methods applied by the 547 current study.

548 Hybridization capture found the greatest number of 5' integration sites, which included 549 nearly all integration sites identified by SPEX and $86.71 \%$ of the integration sites identified by PEC (Figure 5). In contrast, for the 3' LTRs, PEC yielded the most integration sites including $85.07 \%$ and $91.67 \%$ of the integration sites identified by SPEX and hybridization capture respectively. The results were generally consistent across individuals and with the data pooled (Table S6), with no single sample driving the biases for the 5 ' or 3' integration site retrieval thereby validating the reliabity of the methods tested in this study. Considering the output of the methods, the most reliable and comprehensive screening of museum DNA for sequences flanking a target can be achieved by performing PEC and hybridization capture in combination. Both methods covered nearly the full diversity of integration sites identified by SPEX. However, PEC and hybridization capture each retrieved integration sites unique to the method and had reciprocal biases in retrieving 5' and 3' integration sites. It should also be considered that because not all integration sites could be paired for 5' and 3' LTRs, it is clear that not all integration sites present in the samples were retrieved, even when combining all methods. The strong biases towards the 5' or 3' integration sites may prevent such comprehensive analysis from historical samples except at very high sequence coverage depth, for example, using Illumina HiSeq sequencing. identical target site duplications identified 63 matches using the wallaby genome as a reference.

567 The success rate would likely improve upon the availability of an assembled koala reference 
568 genome. Genome data available to this project was represented by unassembled raw reads of

$56998 \mathrm{bp}$ average length. Among the $63 \mathrm{KoRV}$ integration sites identified by this method, 40 were

570 identified after concatenating 5' and 3' flanks derived from the same individual koala. A total of

57123 integration sites were identified by querying with a sequence that concatenated 5' and 3'

572 flanking sites from different koala individuals. This result demonstrates that although many

573 integration sites were identified per koala, they were not identified comprehensively and many

574 integration sites were missed. Considering that there are an estimated $165 \mathrm{KoRV}$ copies per

575 haploid genome in Queensland koalas (Tarlinton, Meers \& Young, 2006), exhaustive

576 identification of integration sites would have required detection of 1,650 5' and 3' integration

577 sites across the 10 koalas used in the study. Moreover, for aDNA, comprehensive identification

578 of integration sites is even more challenging due to the poor and variable condition of the

579 samples, which results in a decrease in the number of endogenous DNA copies.

580

Little sharing of integration sites between museum samples in this study and those in in Tsangaras et al. 2014 (Tsangaras et al., 2014b) were found (none at 3' and three at 5'). This is

582

583

584

585

586

587

588

589

590

591

592

593

594

595

596

597

possibly due to the methodology difference between the two studies: in Tsangaras et al. 2014, the integration sites and the ends of KoRV LTRs were intentionally avoided for targeted hybridization capture retrieval of KoRV proviral sequences. The integration sites in this same study were captured due to the high CapFlank (Tsangaras et al., 2014a) nature of hybridization capture. In contrast, our study specifically focused on targeted retrieval of integration sites, which were more intensively studied using three techniques. Ishida et al. 2015 (Ishida et al., 2015) also used a different technical strategy than this study, namely genome walking. The focus of their study, like our own, was also integration site retrieval. This is evidenced by a slightly higher number of integration sites shared between museum koalas of our study and modern koalas in Ishida et al. 2015.

Generally, the low number of shared integration sites between the three studies can be due to the varying level of intensiveness for KoRV flank retrieval, which can potentially miss many shared integration sites. Given the independent aims and methods used across the three studies, statistical modeling of shared KoRV integration sites through time was only performed for the ten museum koalas in this study (Article S1 Fig S2). While the number of koalas examined is few, a statistically significant increase in integration site sharing was observed over 
598 time. This could be explained by increased fixation of KoRVs over time. However, with only ten 599 samples, regional differences in fixation of KoRVs e.g. the three young koalas from NSW could 600 also explain the trend as a geographic rather than temporal trend. The methods applied in the 601 current study should allow for a broader screen of museum koalas to distinguish between these 602 possibilities. However, the current study confirms that in general, koalas share few integration 603 sites among individuals in Queensland where KoRV is ubiquitous which contrasts with most 604 known ERVs which are either fixed in the genome of the host species or are at very high 605 frequency. This is further evidence that the KoRV invasion of the koala genome is still in the 606 early stages.

607

608

609

610

611

612

613

614

615

616

617

618

619

620

621

622

623

624

Acknowledgments

625 The authors thank Jörns Fickel for computational support. The authors also thank Joachim Selbig 626 and his staff in the Research Group of Bioinformatics, Institute for Biochemistry and Biology, 
627 University of Potsdam. For museum specimens, we thank F. Johansson and G. Nilson

628 (Bohusläns Museum), R. Timm (Natural History Museum - University of Kansas), J. Chupasko

629 and H. Hoekstra (Harvard Museum of Comparative Zoology), W. Longmore (Museum Victoria),

630 O. Grönwall and U. Johansson (Swedish Natural History Museum), J. Eger (Royal Ontario

631 Museum), S. Hinshaw (University of Michigan Museum of Zoology), D. Stemmer and C.

632 Kemper (South Australian Museum), S. Ingleby (Australian Museum), S. Van Dyck and H.

633 Janetzki (Queensland Museum). The content is solely the responsibility of the authors and does

634 not necessarily represent the official vies of the NIGMS or the National Institutes of Health.

\section{REFERENCES}

636 Allentoft ME., Collins M., Harker D., Haile J., Oskam CL., Hale ML., Campos PF., Samaniego JA., Gilbert MTP., Willerslev E., Zhang G., Scofield RP., Holdaway RN., Bunce M. 2012. The half-life of DNA in bone: measuring decay kinetics in 158 dated fossils. Proceedings. Biological Sciences / The Royal Society 279:4724-4733.

Altschul SF., Gish W., Miller W., Myers EW., Lipman DJ. 1990. Basic local alignment search tool. Journal of molecular biology 215:403-410.

642 Ávila-Arcos MC., Ho SYW., Ishida Y., Nikolaidis N., Tsangaras K., Hönig K., Medina R., KM., Roca AL., Greenwood AD. 2013. One hundred twenty years of koala retrovirus

Blikstad V., Benachenhou F., Sperber GO., Blomberg J. 2008. Evolution of human endogenous evolution determined from museum skins. Molecular biology and evolution 30:299-304. retroviral sequences: a conceptual account. Cellular and molecular life sciences: CMLS 65:3348-3365.

Bolger AM., Lohse M., Usadel B. 2014. Trimmomatic: A flexible trimmer for Illumina 
651 Briggs AW., Good JM., Green RE., Krause J., Maricic T., Stenzel U., Pääbo S. 2009. Primer extension capture: targeted sequence retrieval from heavily degraded DNA sources. Journal of Visualized Experiments: JoVE:1573.

Bromham L. 2002. The human zoo: endogenous retroviruses in the human genome. Trends in 655 Ecology \& Evolution 17:91-97.

656

Brotherton P., Endicott P., Sanchez JJ., Beaumont M., Barnett R., Austin J., Cooper A. 2007. Novel high-resolution characterization of ancient DNA reveals C $>$ U-type base modification events as the sole cause of post mortem miscoding lesions. Nucleic Acids Research 35:5717-5728.

Bushman F., Lewinski M., Ciuffi A., Barr S., Leipzig J., Hannenhalli S., Hoffmann C. 2005. Genome-wide analysis of retroviral DNA integration. Nature Reviews. Microbiology $3: 848-858$.

Cereseto A., Giacca M. 2004. Integration site selection by retroviruses. AIDS reviews 6:13-21.

Chen Y., Yao H., Thompson EJ., Tannir NM., Weinstein JN., Su X. 2012. VirusSeq: Software to identify viruses and their integration sites using nextgeneration sequencing of human cancer tissue. Bioinformatics:bts665.

Ciuffi A., Barr SD. 2011. Identification of HIV integration sites in infected host genomic DNA. Methods (San Diego, Calif.) 53:39-46.

Coffin JM., Hughes SH., Varmus HE. (eds.) 1997. Retroviruses. Cold Spring Harbor (NY): Cold Spring Harbor Laboratory Press.

Der Sarkissian C., Allentoft ME., Ávila-Arcos MC., Barnett R., Campos PF., Cappellini E., Ermini L., Fernández R., da Fonseca R., Ginolhac A., Hansen AJ., Jónsson H., Korneliussen T., Margaryan A., Martin MD., Moreno-Mayar JV., Raghavan M., 
674

675

676

677

678

679

680

681

682

683

684

685

686

687

688

689

690

691

692

693

694

695

Rasmussen M., Velasco MS., Schroeder H., Schubert M., Seguin-Orlando A., Wales N., Gilbert MTP., Willerslev E., Orlando L. 2015. Ancient genomics. Philosophical

Transactions of the Royal Society of London. Series B, Biological Sciences 370:20130387.

Duncavage EJ., Magrini V., Becker N., Armstrong JR., Demeter RT., Wylie T., Abel HJ., Pfeifer

JD. 2011. Hybrid Capture and Next-Generation Sequencing Identify Viral Integration

Sites from Formalin-Fixed, Paraffin-Embedded Tissue. The Journal of Molecular

Diagnostics : JMD 13:325-333.

Enright AJ., Van Dongen S., Ouzounis CA. 2002. An efficient algorithm for large-scale detection of protein families. Nucleic Acids Research 30:1575-1584.

Fields BN., Knipe DM., Howley PM. (eds.) 1996. Fields virology. Philadelphia: LippincottRaven Publishers.

Gifford R., Tristem M. 2003. The evolution, distribution and diversity of endogenous retroviruses. Virus Genes 26:291-315.

Gilbert MTP., Tomsho LP., Rendulic S., Packard M., Drautz DI., Sher A., Tikhonov A., Dalén L., Kuznetsova T., Kosintsev P., Campos PF., Higham T., Collins MJ., Wilson AS., Shidlovskiy F., Buigues B., Ericson PGP., Germonpré M., Götherström A., Iacumin P., Nikolaev V., Nowak-Kemp M., Willerslev E., Knight JR., Irzyk GP., Perbost CS., Fredrikson KM., Harkins TT., Sheridan S., Miller W., Schuster SC. 2007. WholeGenome Shotgun Sequencing of Mitochondria from Ancient Hair Shafts. Science 317:1927-1930.

Hüser D., Gogol-Döring A., Lutter T., Weger S., Winter K., Hammer E-M., Cathomen T., Reinert K., Heilbronn R. 2010. Integration Preferences of Wildtype AAV-2 for 
6.

698

699

700

701

702

703

704

705

706

707

708

709

710

711

712

713

714

715

716

Ishida Y., Zhao K., Greenwood AD., Roca AL. 2015. Proliferation of endogenous retroviruses in the early stages of a host germ line invasion. Molecular Biology and Evolution 32:109120.

Katoh K., Misawa K., Kuma K., Miyata T. 2002. MAFFT: a novel method for rapid multiple sequence alignment based on fast Fourier transform. Nucleic Acids Research 30:30593066.

Kearse M., Moir R., Wilson A., Stones-Havas S., Cheung M., Sturrock S., Buxton S., Cooper A., Markowitz S., Duran C., Thierer T., Ashton B., Meintjes P., Drummond A. 2012. Geneious Basic: an integrated and extendable desktop software platform for the organization and analysis of sequence data. Bioinformatics (Oxford, England) 28:16471649.

Khodosevich K., Lebedev Y., Sverdlov E. 2002. Endogenous Retroviruses and Human Evolution. Comparative and Functional Genomics 3:494-498.

Kircher M., Sawyer S., Meyer M. 2012. Double indexing overcomes inaccuracies in multiplex sequencing on the Illumina platform. Nucleic Acids Research 40:e3.

Kustikova OS., Modlich U., Fehse B. 2009. Retroviral insertion site analysis in dominant haematopoietic clones. Methods in Molecular Biology (Clifton, N.J.) 506:373-390.

Kvaratskhelia M., Sharma A., Larue RC., Serrao E., Engelman A. 2014. Molecular mechanisms of retroviral integration site selection. Nucleic Acids Research:gku769. 
717 LaFave MC., Varshney GK., Gildea DE., Wolfsberg TG., Baxevanis AD., Burgess SM. 2014.

718 MLV integration site selection is driven by strong enhancers and active promoters.

$719 \quad$ Nucleic Acids Research:gkt1399.

720 Lewinski MK., Yamashita M., Emerman M., Ciuffi A., Marshall H., Crawford G., Collins F.,

721 Shinn P., Leipzig J., Hannenhalli S., Berry CC., Ecker JR., Bushman FD. 2006.

722 Retroviral DNA Integration: Viral and Cellular Determinants of Target-Site Selection.

$723 \quad$ PLoS Pathogens 2.

724 Li W., Jaroszewski L., Godzik A. 2001. Clustering of highly homologous sequences to reduce 725 the size of large protein databases. Bioinformatics 17:282-283.

726 Magoč T., Salzberg SL. 2011. FLASH: Fast Length Adjustment of Short Reads to Improve 727 Genome Assemblies. Bioinformatics:btr507.

728 Maricic T., Whitten M., Pääbo S. 2010. Multiplexed DNA Sequence Capture of Mitochondrial 729 Genomes Using PCR Products. PLoS ONE 5:e14004.

730 Martin M. 2011. Cutadapt removes adapter sequences from high-throughput sequencing reads.

$731 \quad$ EMBnet.journal 17:pp. 10-12.

732 Meredith RW., Westerman M., Springer MS. 2009. A phylogeny of Diprotodontia (Marsupialia)

733 based on sequences for five nuclear genes. Molecular Phylogenetics and Evolution

$734 \quad 51: 554-571$.

735 Meyer M., Briggs AW., Maricic T., Höber B., Höffner B., Krause J., Weihmann A., Pääbo S.,

736 Hofreiter M. 2008. From micrograms to picograms: quantitative PCR reduces the

737 material demands of high-throughput sequencing. Nucleic Acids Research 36:e5.

738 Meyer M., Kircher M. 2010. Illumina Sequencing Library Preparation for Highly Multiplexed

739 Target Capture and Sequencing. Cold Spring Harbor Protocols 2010:pdb.prot5448. 
740 Mitchell RS., Beitzel BF., Schroder ARW., Shinn P., Chen H., Berry CC., Ecker JR., Bushman

741 FD. 2004. Retroviral DNA integration: ASLV, HIV, and MLV show distinct target site $742 \quad$ preferences. PLoS biology 2:E234.

743 Moalic Y., Blanchard Y., Félix H., Jestin A. 2006. Porcine Endogenous Retrovirus Integration

744 Sites in the Human Genome: Features in Common with Those of Murine Leukemia Virus.

$745 \quad$ Journal of Virology 80:10980-10988.

746 Nowrouzi A., Dittrich M., Klanke C., Heinkelein M., Rammling M., Dandekar T., von Kalle C., Rethwilm A. 2006. Genome-wide mapping of foamy virus vector integrations into a human cell line. The Journal of General Virology 87:1339-1347.

Pääbo S., Poinar H., Serre D., Jaenicke-Despres V., Hebler J., Rohland N., Kuch M., Krause J., Vigilant L., Hofreiter M. 2004. Genetic analyses from ancient DNA. Annual Review of

Pontius JU., Mullikin JC., Smith DR., Lindblad-Toh K., Gnerre S., Clamp M., Chang J., Stephens R., Neelam B., Volfovsky N., Schäffer AA., Agarwala R., Narfström K., Murphy WJ., Giger U., Roca AL., Antunes A., Menotti-Raymond M., Yuhki N., PeconSlattery J., Johnson WE., Bourque G., Tesler G., O’Brien SJ. 2007. Initial sequence and

Renfree MB., Papenfuss AT., Deakin JE., Lindsay J., Heider T., Belov K., Rens W., Waters PD., 758 Pharo EA., Shaw G., Wong ESW., Lefèvre CM., Nicholas KR., Kuroki Y., Wakefield MJ., Zenger KR., Wang C., Ferguson-Smith M., Nicholas FW., Hickford D., Yu H., Short KR., Siddle HV., Frankenberg SR., Chew KY., Menzies BR., Stringer JM., Suzuki S., Hore TA., Delbridge ML., Patel HR., Mohammadi A., Schneider NY., Hu Y., O’Hara W., Al Nadaf S., Wu C., Feng Z-P., Cocks BG., Wang J., Flicek P., Searle SMJ., Fairley 

Wood D., Williams S., Sundaravadanam Y., Muzny DM., Jhangiani SN., Lewis LR., Morgan MB., Okwuonu GO., Ruiz SJ., Santibanez J., Nazareth L., Cree A., Fowler G., Kovar CL., Dinh HH., Joshi V., Jing C., Lara F., Thornton R., Chen L., Deng J., Liu Y.,

Rice P., Longden I., Bleasby A. 2000. EMBOSS: the European Molecular Biology Open Shen JY., Song X-Z., Edson J., Troon C., Thomas D., Stephens A., Yapa L., Levchenko

Roca AL., Ishida Y., Nikolaidis N., Kolokotronis S-O., Fratpietro S., Stewardson K., Hensley S., T., Gibbs RA., Cooper DW., Speed TP., Fujiyama A., Graves JAM., O’Neill RJ., Pask AJ., Forrest SM., Worley KC. 2011. Genome sequence of an Australian kangaroo, Macropus eugenii, provides insight into the evolution of mammalian reproduction and development. Genome Biology 12:R81. Tisdale M., Boeskorov G., Greenwood AD. 2009. Genetic variation at hair length candidate genes in elephants and the extinct woolly mammoth. BMC Evolutionary Biology 9:232.

Rousset F., Ferdy J-B. 2014. Testing environmental and genetic effects in the presence of spatial autocorrelation. Ecography 37:781-790. within the chicken population. Journal of Virology 75:11935-11938.

784 Santoni FA., Hartley O., Luban J. 2010. Deciphering the code for retroviral integration target site 785 selection. PLoS computational biology 6:e1001008. 
786 Schmidt M., Schwarzwaelder K., Bartholomae C., Zaoui K., Ball C., Pilz I., Braun S., Glimm H.,

787 von Kalle C. 2007. High-resolution insertion-site analysis by linear amplification-

788 mediated PCR (LAM-PCR). Nature Methods 4:1051-1057.

789 Simmons GS., Young PR., Hanger JJ., Jones K., Clarke D., McKee JJ., Meers J. 2012. Prevalence of koala retrovirus in geographically diverse populations in Australia. Australian Veterinary Journal 90:404-409.

792 Stajich., Block D., Boulez K., Brenner SE., Chervitz SA., Dagdigian C., Fuellen G., Gilbert JGR., 793 Korf I., Lapp H., others. 2002. The Bioperl toolkit: Perl modules for the life sciences.

Tarlinton R., Meers J., Hanger J., Young P. 2005. Real-time reverse transcriptase PCR for the endogenous koala retrovirus reveals an association between plasma viral load and neoplastic disease in koalas. The Journal of General Virology 86:783-787.

Tarlinton RE., Meers J., Young PR. 2006. Retroviral invasion of the koala genome. Nature 799 442:79-81.

800 Tarlinton R., Meers J., Young P. 2008. Biology and evolution of the endogenous koala retrovirus. $801 \quad$ Cellular and molecular life sciences: CMLS 65:3413-3421.

802 Tsangaras K., Wales N., Sicheritz-Pontén T., Rasmussen S., Michaux J., Ishida Y., Morand S., 803 Kampmann M-L., Gilbert MTP., Greenwood AD. 2014a. Hybridization capture using 804 short PCR products enriches small genomes by capturing flanking sequences (CapFlank). $805 \quad$ PloS One 9:e109101.

806 Tsangaras K., Siracusa MC., Nikolaidis N., Ishida Y., Cui P., Vielgrader H., Helgen KM., Roca 807 AL., Greenwood AD. 2014b. Hybridization Capture Reveals Evolution and Conservation $808 \quad$ across the Entire Koala Retrovirus Genome. PLoS ONE 9:e95633. 
809 Wang Q., Jia P., Zhao Z. 2013. VirusFinder: software for efficient and accurate detection of

810 viruses and their integration sites in host genomes through next generation sequencing 811 data. PloS One 8:e64465.

812 Waterhouse AM., Procter JB., Martin DMA., Clamp M., Barton GJ. 2009. Jalview Version 2--a

813 multiple sequence alignment editor and analysis workbench. Bioinformatics (Oxford,

$814 \quad$ England) 25:1189-1191.

815 Willerslev E., Cooper A. 2005. Review Paper. Ancient DNA. Proceedings of the Royal Society

816 of London B: Biological Sciences 272:3-16.

817 Wragg D., Mason AS., Yu L., Kuo R, Lawal1 RA, Desta TT., Mwacharo JM., Cho C., Kemp S.,

818 Burt DW., Hanotte O. 2015. Genome-wide analysis reveals the extent of EAV-HP

819 integration in domestic chicken. BMC Genomic 16: 784Wyatt KB., Campos PF., Gilbert

820 MTP., Kolokotronis S-O., Hynes WH., DeSalle R., Daszak P., MacPhee RDE.,

821 Greenwood AD. 2008. Historical Mammal Extinction on Christmas Island (Indian Ocean)

822 Correlates with Introduced Infectious Disease. PLoS ONE 3:e3602.

823

824 Figure legends

825 Figure 1. Experimental work flow for the three enrichment techniques.

826 Abbreviations: HC for Hybridization Capture; PEC for Primer Extension Capture; SPEX

827 for Single Primer Extension. A square of $7 \mathrm{~mm}$ x $7 \mathrm{~mm}$ of koala skin tissue per museum specimen 828 was extracted in a dedicated ancient DNA (aDNA) facility. A workflow for the three techniques 829 is illustrated. Both $\mathrm{HC}$ and PEC require Illumina library preparation as a preliminary step. The 830 double stranded libraries are denatured to single stranded DNA molecules and underwent 831 different experimental procedures in $\mathrm{HC}$ and PEC. In HC, single stranded DNA libraries are 832 mixed with magnetic beads immobilized with baits. These are incubated by slow rotation at $65^{\circ} \mathrm{C}$ 833 for 48 hours. After a series of wash steps, the libraries with non-targets sequences are washed off 
834 leaving only the libraries with target sequences hybridized with the baits on beads. These target 835 molecules were then dissociated from the baits using a special elution buffer, and were used as 836 templates for PCR amplification. While in PEC, the singled stranded libraries are mixed with 837 biotinylated oligos for 1 minute at $55^{\circ} \mathrm{C}$ in which only the libraries with target sequences 838 hybridized with the biotinylated oligos. Primer extension reactions of the biotinylated oligos 839 were performed only to these hybridized libraries. Biotinylated oligos were collected by 840 magnetic beads together with the hybridized targeted libraries. The single stranded libraries with 841 target sequences were dissociated with biotinylated oligos and were eluted for subsequent PCR 842 amplification. In contrast for SPEX, DNA extracts are directly denatured to be single stranded 843 and mixed with the same biotinylated oligos used in PEC for 1 minute at $55^{\circ} \mathrm{C}$. Similar as in 844 PEC, primer extension reactions of the biotinylated oligos were performed only to the single 845 molecules (target sequences) hybridized with biotinylated oligos. These hybrid molecules were 846 collected using magnetic beads. The original single stranded target molecules were washed away 847 and the biotinylated oligos with $3^{\prime}$ extension were eluted off the beads and were treated with a 848 poly $\mathrm{C}$ tailing reaction. These poly $\mathrm{C}$ tailed molecules were amplified using primers with a $5^{\prime}$ 849 overhang of the Illumina sequencing adaptor. Through this process, the SPEX products were 850 constructed into Illumina libraries without an additional library preparation step. These SPEX851 Illumina libraries were then used in an index PCR and a further amplification step. As shown, 852 SPEX requires at least one more amplification step than HC or PEC, which may explain the high 853 level of clonality in the SPEX result. 
855 Figure 2. Experimental design for the identification of KoRV integration sites.

856 Panel A illustrates that the genome of the koala retrovirus (KoRV) has two identical long 857 terminal repeats (LTRs) on both ends. The primers or baits can bind to both LTRs, so two 858 categories of products exist: A) products extending into the flanks from primer extension a; B) 859 products extending into the middle of KoRV genome from primer extension $b$. In principle, there 860 should be an equal number of sequences for the two categories. Panel B indicates that the KoRV 861 LTRs contain three components, U3, R and U5. For SPEX, primers were partially nested. All 862 primers are $20 \mathrm{bp}$ long, and there is a 8 bp-overlap between the inner primers (3.1 and 5.1) and 863 outer primers (3.2 and 5.2) respectively. To avoid known polymorphisms in the LTR, the 3' end 864 of outer primers are $17 \mathrm{bp}$ from the 5' end of LTR and $50 \mathrm{bp}$ from the 3' end of LTR. Since the 5' 865 LTR and 3' LTR of the same KoRV are identical products can also extend into the KoRV 866 genome. The 5' and 3' flanks can be distinguished by their linked LTR end, with the 5' flank 867 linked to 5' LTR and 3' flank linked to 3' LTR. Considering the longest deletion found at the 868 end of LTR is $19 \mathrm{bp}$, the LTR end was divided into two segments for subsequent computational 869 identification: the B region representing the last $19 \mathrm{bp}$ of the LTR, and the A region representing 870 the rest of LTR end.

871 Figure 3. Bioinformatic pipeline for identification of KoRV integration sites.

872 The pipeline was run separately for each data set obtained by three different techniques.

873 For the key steps, the number of sequences retained is indicated in parentheses for each 874 technique in this order from left to right: PEC, SPEX and hybridization capture. After processing 875 NGS reads, KoRV integration sites were identified in a two-step analysis of KoRV LTR ends, 876 next to the host DNA flanking KoRV. The first round of selection targeted the A region of the 877 LTR end and its output, was used for subsequent identification of the B region. The LTR ends of 878 all sequences were trimmed off, and only sequences longer than four bp were considered. Using 879 a sequence clustering approach, unique vs. shared integration sites were sorted into clusters. The 880 consensus of each non-singleton cluster was computed using a multiple sequence alignment.

881 These consensus sequences and singleton sequences were queried against wallaby genomic 882 scaffolds and koala Illumina Hiseq reads to determine whether they represented KoRV flanking 883 sequences. At the same time extension products into the KoRV genome were identified. 
884 Figure 4. Pairing of 5' and 3' integration site flanking sequences.

885 The first 4 bp beyond the KoRV 5' LTR is the target site duplication (e.g. ACAT in this 886 figure), and the same $4 \mathrm{bp}$ is found at the beginning of a 3' flank (Ishida et al. 2015). One copy 887 of the target site duplication was trimmed off and the two flanks were concatenated. The paired 5'888 3' integration sites were then screened against the wallaby draft genome and koala Hiseq 889 genomic sequences.

890 Figure 5. Venn diagrams of KoRV integration sites found by different methods.

891 For 5' integration sites, HC (hybridization capture) yielded the highest total number of 892 integration sites (887), and covered $91.3 \%$ of the integration sites found by SPEX and $86.7 \%$ of 893 the integration sites found by PEC. For 3' integration sites, PEC yielded the highest total 894 number of integration sites (672), and covered $81.4 \%$ of the integration sites found by SPEX and $89591.7 \%$ of the integration sites found by hybridization capture.For the retrieval of both 5' and 3' 896 integration sites, SPEX showed the worst performance (smallest number of integration sites 897 retrieved among three enrichment methods).

898 Figure 6. Target sequences distribution of three techniques.

899 Abbreviations: HC for Hybridization Capture; PEC for Primer Extension Capture; SPEX 900 for Single Primer Extension. The oligos used for all three experiments bind near to the end 901 region of the KoRV LTR. Because the genome of KoRV has two identical long terminal repeats 902 (LTRs) on both ends, primer extension of captured products using these oligos will yield two 903 categories of products; (i) KoRV flanks, the desired products for this study which extend into the 904 koala DNA flanking KoRV and (ii) Internal KoRV reads, sequences extending towards the 905 middle of KoRV genome. The bold black line at the bottom of each technical section 906 approximately present the target sequences in the final result, showing a bias towards the $3^{\prime}$ end 907 for PEC and a bias towards 5' for HC.

908 ADDITIONAL FILES

909 Table S1. Indexing primers for Illumina library construction. The Illumina indices (6-7 bp

910 long) are embedded in the primers as indicated in lower case. 
911 Table S2. Primers used in our experiments for PEC, SPEX and to generate bait for

912 hybridization capture.

913 Table S3. Information on all koala samples compared in this study.

914 Table S4. KoRV 3' integration sites shared among at least two koalas. Koala samples are

915 listed from left to right according to sampling age (with the oldest samples on the left). The

916 integration sites from this study, Tsangaras et al. 2014 and Ishida et al. 2015 were compared.

917 Table S5. KoRV 5' integration sites shared among at least two koalas. Koala samples are

918 listed from left to right according to sampling age (with the oldest samples on the left). The

919 integration sites from this study, Tsangaras et al. 2014 (Tsangaras et al., 2014b) and Ishida et al.

9202015 (Ishida et al., 2015) were compared.

921 Table S6. Sequence filtering results. Illumina Miseq 150 bp Pair End run results are shown

922 with R1 and R2 indicating the forward and reverse reads of sequencing. Reductions of retained

923 sequences are shown for each step of the bioinformatic pipeline.

924 Fig. S1: Length distribution of raw sequence reads. $\mathrm{R}$ plot of sequence length distributions

925 from PEC (red), SPEX (blue) and hybridization capture (HC) (green) reads.

926 Fig. S2: The proportion of KoRV integration sites that are shared among koalas may be

927 increasing over time. Plotted results of a Generalised Mixed effect Model analysis against the

928 shared integration sites by year.

929 Data S1: Matched 5-3 integration site from the same koala. The sequences shown are the

930 concatenation of matched 5' and 3' integration sites from a single koala individual.

931 Data S2: Matched 5-3 integration site from different koalas. The sequences shown are the

932 concatenation of matched 5' and 3' integration sites from different koala individuals.

933 Data S3: Scripts developed specifically for the integration site identification pipeline.

934 Additional scripts not developed in this study are referenced in the text and only novel scripts

935 developed for this study are included.

936 Article S1: Supplementary results and discussion on statistical modeling analysis. A

937 statistical analysis was performed on shared KoRV integration sites among our 10 koala samples.

938 The results of this analysis are described and discussed.

939 


\section{Figure 1 (on next page)}

\section{Figure 1. Experimental work flow for thethree enrichment techniques}

Figure 1. Experimental work flow for the three enrichment techniques.

Abbreviations: HC for Hybridization Capture; PEC for Primer Extension Capture; SPEX for Single Primer Extension. A square of $7 \mathrm{~mm} \times 7 \mathrm{~mm}$ of koala skin tissue per museum specimen was extracted in a dedicated ancient DNA (aDNA) facility. A workflow for the three techniques is illustrated. Both HC and PEC require Illumina library preparation as a preliminary step. The double stranded libraries are denatured to single stranded DNA molecules and underwent different experimental procedures in HC and PEC. In HC, single stranded DNA libraries are mixed with magnetic beads immobilized with baits. These are incubated by slow rotation at $65^{\circ} \mathrm{C}$ for 48 hours. After a series of wash steps, the libraries with non-targets sequences are washed off leaving only the libraries with target sequences hybridized with the baits on beads. These target molecules were then dissociated from the baits using a special elution buffer, and were used as templates for PCR amplification. While in PEC, the singled stranded libraries are mixed with biotinylated oligos for 1 minute at $55^{\circ} \mathrm{C}$ in which only the libraries with target sequences hybridized with the biotinylated oligos. Primer extension reactions of the biotinylated oligos were performed only to these hybridized libraries. Biotinylated oligos were collected by magnetic beads together with the hybridized targeted libraries. The single stranded libraries with target sequences were dissociated with biotinylated oligos and were eluted for subsequent PCR amplification. In contrast for SPEX, DNA extracts are directly denatured to be single stranded and mixed with the same biotinylated oligos used in PEC for 1 minute at $55^{\circ} \mathrm{C}$. Similar as in PEC, primer extension reactions of the biotinylated oligos were performed only to the single molecules (target sequences) hybridized with biotinylated oligos. These hybrid molecules were collected using magnetic beads. The original single stranded target molecules were washed away and the biotinylated oligos with 3' extension were eluted off the beads and were treated with a poly $\mathrm{C}$ tailing reaction. These poly $\mathrm{C}$ tailed molecules were amplified using primers with a $5^{\prime}$ overhang of the Illumina sequencing adaptor. Through this process, the SPEX products were constructed into Illumina libraries without an additional library preparation step. These SPEX-Illumina libraries were then used in an index PCR and a further amplification step. As shown, SPEX requires at least one more amplification step than HC or PEC, which may explain the high level of clonality in the SPEX result. 


\section{Figure 2 (on next page)}

Figure 2. Experimental design for the identification of KoRV integration sites

Figure 2. Panel A illustrates that the genome of the koala retrovirus (KoRV) has two identical long terminal repeats (LTRs) on both ends. The primers or baits can bind to both LTRs, so two categories of products exist: A) products extending into the flanks from primer extension $a$; B) products extending into the middle of KoRV genome from primer extension b. In principle, there should be an equal number of sequences for the two categories. Panel B indicates that the KoRV LTRs contain three components, U3, R and U5. For SPEX, primers were partially nested. All primers are $20 \mathrm{bp}$ long, and there is a 8 bp-overlap between the inner primers ( 3.1 and 5.1) and outer primers (3.2 and 5.2) respectively. To avoid known polymorphisms in the LTR, the 3' end of outer primers are $17 \mathrm{bp}$ from the 5' end of LTR and $50 \mathrm{bp}$ from the 3' end of LTR. Since the 5' LTR and 3' LTR of the same KoRV are identical products can also extend into the KoRV genome. The 5' and 3' flanks can be distinguished by their linked LTR end, with the 5' flank linked to 5' LTR and 3' flank linked to 3' LTR. Considering the longest deletion found at the end of LTR is $19 \mathrm{bp}$, the LTR end was divided into two segments for subsequent computational identification: the B region representing the last $19 \mathrm{bp}$ of the $L T R$, and the A region representing the rest of LTR end. 
(A) 5' primer extension a

5' LTR end with extension into KoRV flanks

\section{5 ' primer extension b}

5' LTR end with extension into KoRV genome

\begin{tabular}{|c|c|c|}
\hline 5' flank & 5' LTR & gag \\
& $\longrightarrow \begin{array}{l}\text { 3' LTR end with extension } \\
\text { into KoRV genome }\end{array}$
\end{tabular}

3' primer extension b

\section{Genome of koala retrovirus (KoRV) 8.4 kb}

(B)

\section{KoRV Long Terminal Repeats (LTRs)}

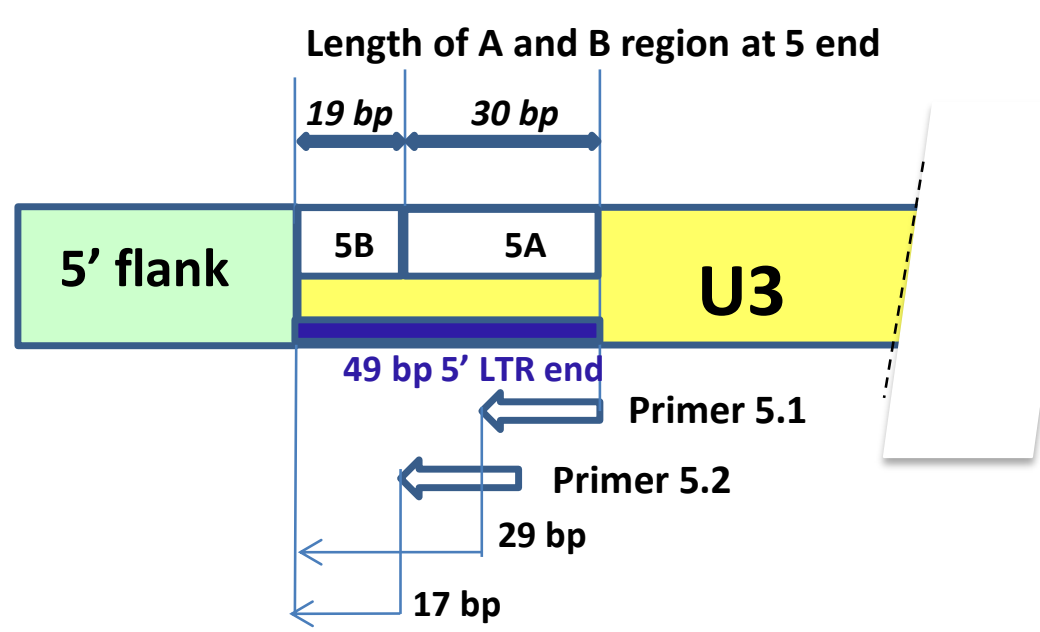

Length of $A$ and $B$ region at 3 end

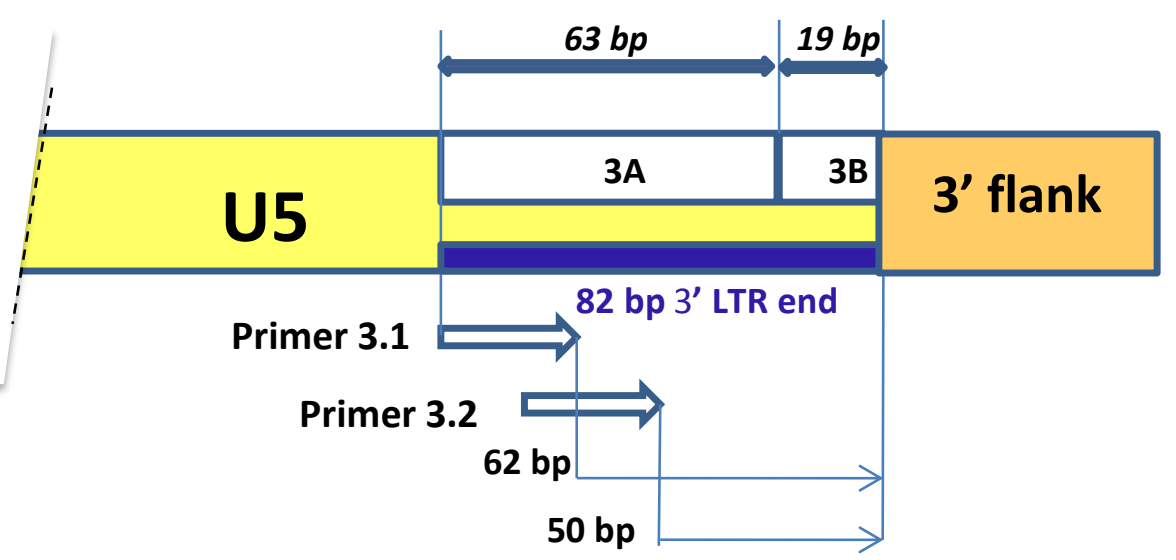




\section{Figure 3 (on next page)}

Figure 3. Bioinformatic pipeline for identification of KoRV integration sites

Figure 3. The pipeline was run separately for each data set obtained by three different techniques. For the key steps, the number of sequences retained is indicated in parentheses for each technique in this order from left to right: PEC, SPEX and hybridization capture. After processing NGS reads, KoRV integration sites were identified in a two-step analysis of KoRV LTR ends, next to the host DNA flanking KoRV. The first round of selection targeted the A region of the LTR end and its output, was used for subsequent identification of the $B$ region. The LTR ends of all sequences were trimmed off, and only sequences longer than four bp were considered. Using a sequence clustering approach, unique vs. shared integration sites were sorted into clusters. The consensus of each non-singleton cluster was computed using a multiple sequence alignment. These consensus sequences and singleton sequences were queried against wallaby genomic scaffolds and koala Illumina Hiseq reads to determine whether they represented KoRV flanking sequences. At the same time extension products into the KoRV genome were identified. 
Raw data

$(7,629,810$ / 6,956,280 / 31,096,064 sequence reads) be reviewed

$\downarrow$

Quality filtering \& preprocessing

(714,929/1,188,365/11,675,245 sequences)

$\downarrow$

Selection of sequences containing region A of LTR

$\downarrow$

Identification of sequences containing region B of LTR

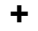

Removal of LTR tails

Selection of hits to wallaby scaffolds or to koala HiSeq reads

Exclusion of short (<4bp) sequences

$$
(15,721 / 2,739 / 1,186 \text { sequences })
$$

$\downarrow$

Clustering of sequences based on sequence similarity

$\downarrow$

Computation of MSA and consensus sequence

for each cluster with $>=2$ sequences

$\downarrow$

Alignment of singletons and consensus

sequences to KoRV genome

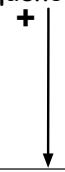

Extension product into

KoRV genome

(435/1,541/165

Peer] reviewing RPE (2016:018693: 19NEW 3 Mar 2016)

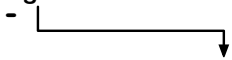

Selection of hits to wallaby scaffolds or to koala HiSeq reads

Extension product into koala genome, i.e., integration site (291 / 815 / 911 sequences) 


\section{Figure 4 (on next page)}

Figure 4. Pairing of $5^{\prime}$ and $3^{\prime}$ integration sites

Figure 4. The first 4 bp beyond the KoRV 5' LTR is the target site duplication (e.g. ACAT in this figure), and the same $4 \mathrm{bp}$ is found at the beginning of a 3' flank (Ishida et al. 2015). One copy of the target site duplication was trimmed off and the two flanks were concatenated. The paired 5'-3' integration sites were then screened against the wallaby draft genome and koala Hiseq genomic sequences. 
Peerd

\begin{tabular}{|l|l|l|l|l|l|l|}
\hline & LTR & gag & pol & env & LTR & \\
\hline
\end{tabular}

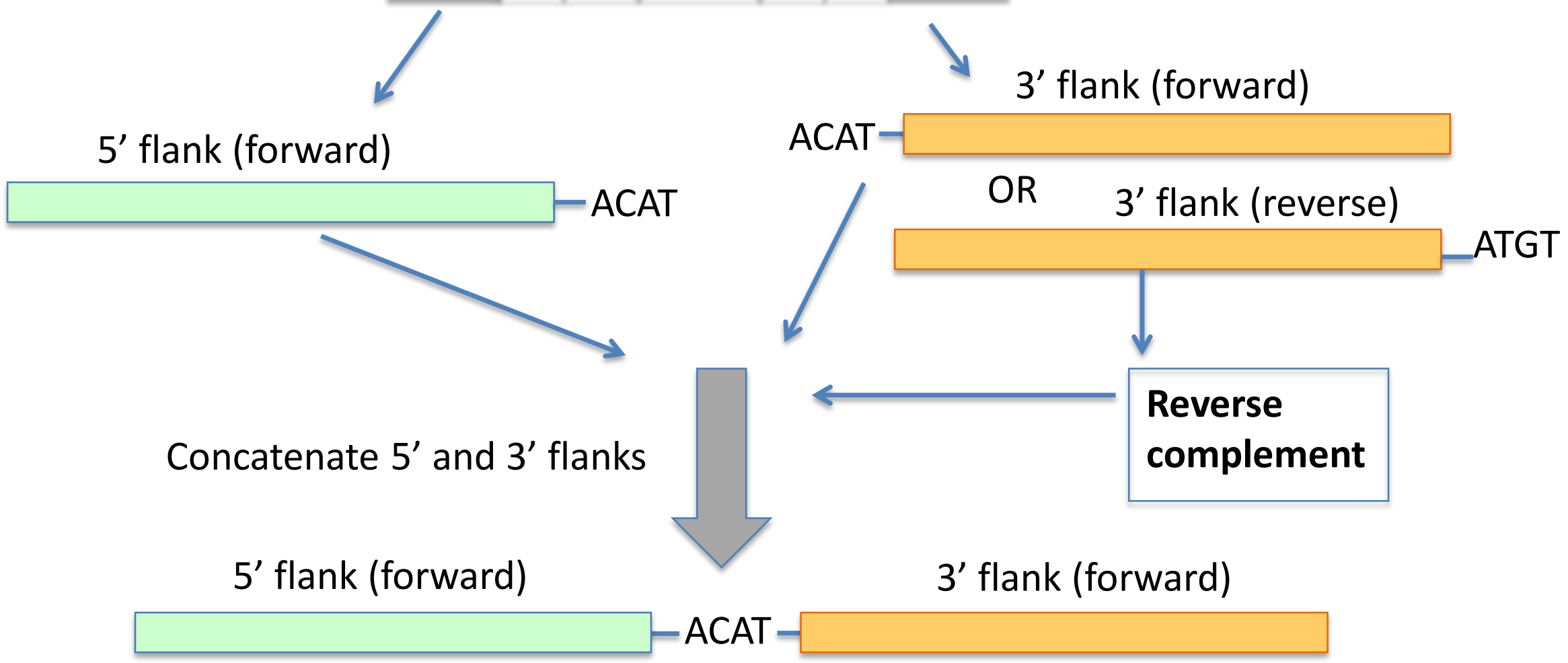

Use concatenated sequence as query

Map to wallaby genome, where KoRV is absent; or BLASTN search against koala Hiseq data 


\section{Figure 5 (on next page)}

Figure 5 . Venn diagrams of KoRV integration sites found by different methods

Figure 5. (A) For 5' integration sites, $\mathrm{HC}$ (hybridization capture) yielded the highest total number of integration sites (887), and covered $91.3 \%$ of the integration sites found by SPEX and $86.7 \%$ of the integration sites found by PEC. (B) For 3' integration sites, PEC yielded the highest total number of integration sites (672), and covered $81.4 \%$ of the integration sites found by SPEX and $91.7 \%$ of the integration sites found by hybridization capture.For the retrieval of both 5' and 3' integration sites, SPEX showed the worst performance (smallest number of integration sites retrieved among three enrichment methods). 


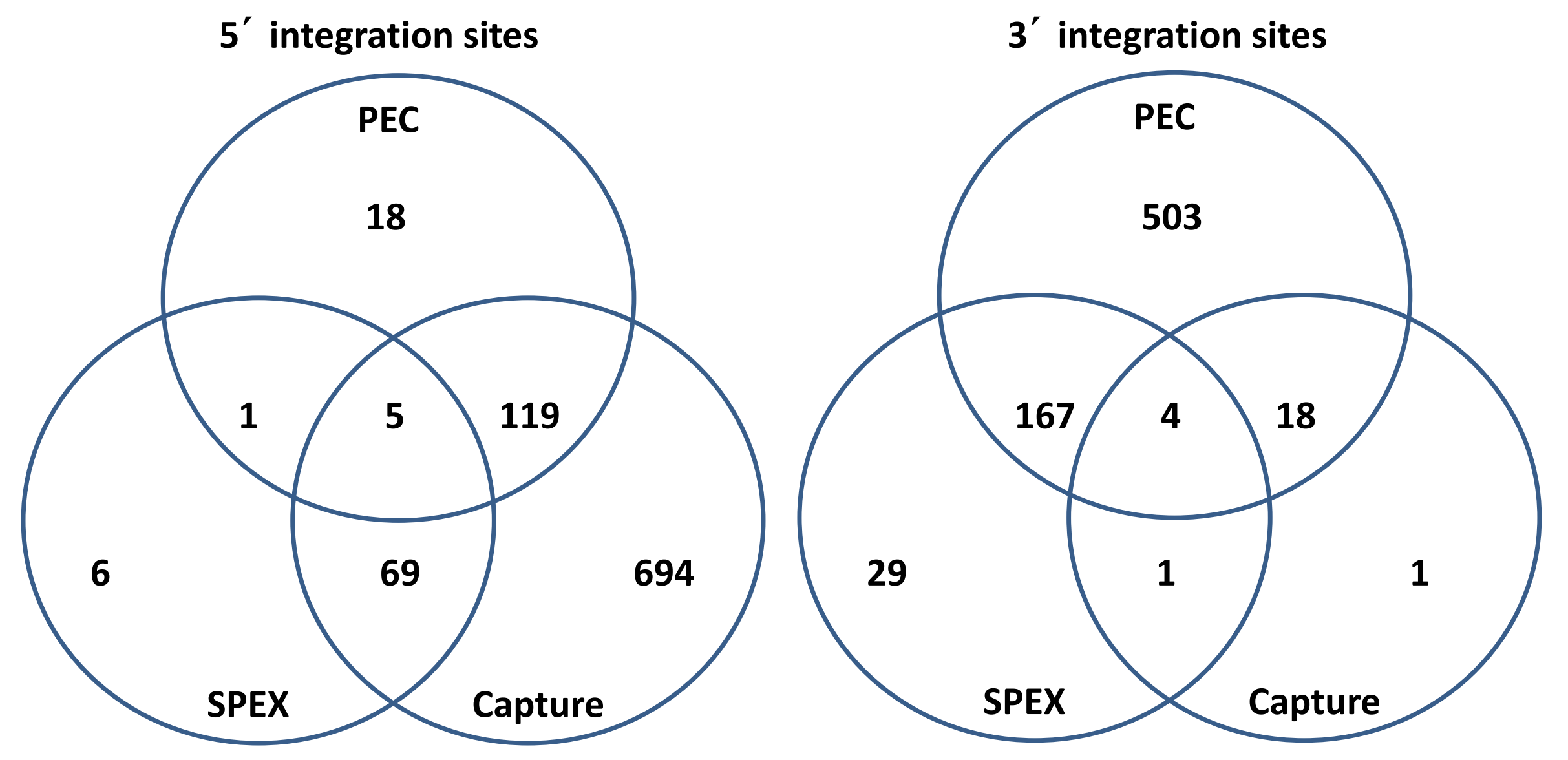




\section{Figure 6 (on next page)}

Figure 6. Target sequences distribution of three techniques

Figure 6. Abbreviations: HC for Hybridization Capture; PEC for Primer Extension Capture; SPEX for Single Primer Extension. The oligos used for all three experiments bind near to the end region of the KoRV LTR. Because the genome of KoRV has two identical long terminal repeats (LTRs) on both ends, primer extension of captured products using these oligos will yield two categories of products; (i) KoRV flanks, the desired products for this study which extend into the koala DNA flanking KoRV and (ii) Internal KoRV reads, sequences extending towards the middle of KoRV genome. The bold black line at the bottom of each technical section approximately present the target sequences in the final result, showing a bias towards the 3' end for PEC and a bias towards 5' for HC. 


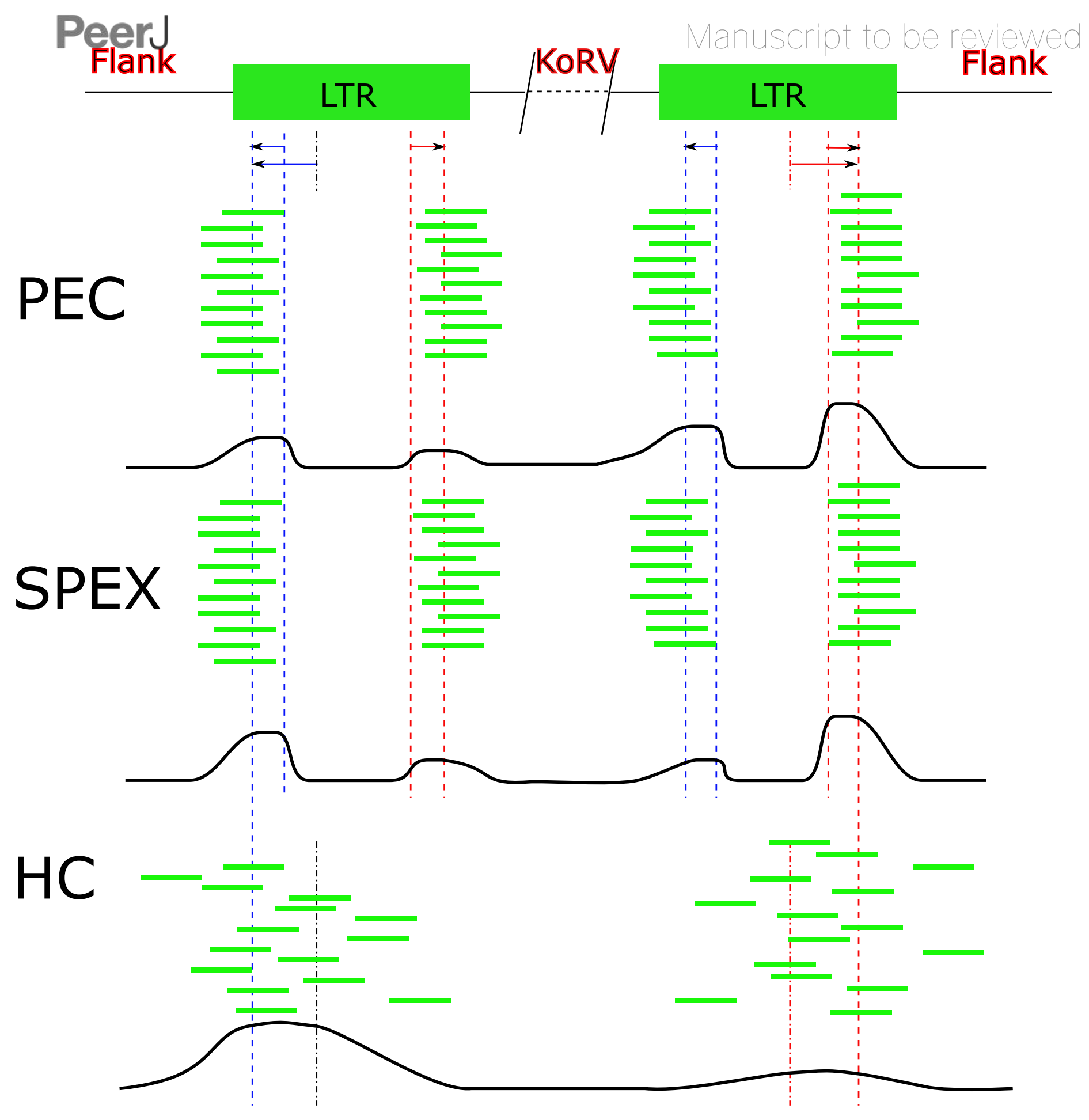




\section{Table $\mathbf{1}$ (on next page)}

Table 1. Koala sample information 


\section{Koala sample information}

\begin{tabular}{|c|c|c|c|c|c|}
\hline ection No. & Sampling Year & Sample provider & Locality & $\begin{array}{l}\text { Number in } \\
\text { experiment }\end{array}$ & PCR res \\
\hline A17300 & 1883 & Australian Museum & New South Wales, Australia; $35^{\circ} 09^{\prime} \mathrm{S}, 149^{\circ} 40^{\prime} \mathrm{E}$ & Koala_1 & Negative \\
\hline IA17311 & 1883 & Australian Museum & New South Wales, Australia; $35^{\circ} 09^{\prime} \mathrm{S}, 149^{\circ} 40^{\prime} \mathrm{E}$ & Koala_2 & Negative \\
\hline IA17299 & 1883 & Australian Museum & New South Wales, Australia; $35^{\circ} 09^{\prime} \mathrm{S}, 149^{\circ} 40^{\prime} \mathrm{E}$ & Koala_3 & Negative \\
\hline U J2377 & 1915 & Queensland Museum & Queensland Australia & Koala_4 & Negative \\
\hline И J7209 & 1945 & Queensland Museum & Queensland Australia & Koala_5 & Positive \\
\hline И J8353 & 1952 & Queensland Museum & Queensland Australia & Koala_6 & Negative \\
\hline JM1875 & 1960s & Queensland Museum & Queensland Australia & Koala_7 & Positive \\
\hline M 12482 & 1971 & Australian Museum & New South Wales, Australia; $33^{\circ} 38^{\prime} \mathrm{S}, 151^{\circ} 20^{\prime} \mathrm{E}$ & Koala_8 & Positive \\
\hline M JM64 & 1973 & Queensland Museum & Queensland Australia & Koala_9 & Positive \\
\hline M 7625 & $1970-1980 s$ & Queensland Museum & Queensland Australia & Koala_10 & $\begin{array}{l}\text { Positive } \\
\text { poorly }\end{array}$ \\
\hline CZ 8574 & 1904 & Museum of Comparative Zoology & Queensland Australia & not sequenced & working \\
\hline 1010180 & 1891 & Royal Ontario Museum & Queensland Australia & not sequenced & Negative \\
\hline 22553 & 1966 & U of Mich Museum of Zoology & Queensland Australia & not sequenced & Negative \\
\hline
\end{tabular}

results for these samples were reported in Avila-Arcos et al. 2013 
Table 2 (on next page)

Table 2. Selection criteria for 2 rounds of pairwise alignment 
Table 2. Selection criteria for 2 rounds of pairwise alignment

Step of Filtering

Segment of LTR tail used for alignment

Length of the LTR tail segment in bp

Minimum alignment length in bp

Minimum identity level in percent 1st pairwise

alignment

$5 \mathrm{~A} \quad 3 \mathrm{~A} \quad 5 \mathrm{~B}$

$30 \quad 63 \quad 19$

$20 \quad 43 \quad 12$

90 2nd pairwise

alignment

B 3B

$19 \quad 19$

$90 \quad 80 \quad 80$

1 


\section{Table 3 (on next page)}

Table 3. Result of analysis for the three technique groups 
Table 3. Result of analysis for the three technique groups

\begin{tabular}{|c|c|c|c|c|c|c|}
\hline \multirow{2}{*}{$\begin{array}{l}\text { Technique } \\
\text { KoRV flanks orientation }\end{array}$} & \multicolumn{2}{|c|}{ SPEX } & \multicolumn{2}{|c|}{ PEC } & \multicolumn{2}{|c|}{ Hybridization Capture } \\
\hline & 5 end & 3 end & 5 end & 3 end & 5 end & 3 end \\
\hline KoRV flanks $<4$ bp & 15822 & 1527 & 496 & 1806 & 191 & 41 \\
\hline KoRV flanks 4-14 & 6426 & 8896 & 329 & 2033 & 1052 & 24 \\
\hline KoRV flanks 15 bp or longer & 95 & 304 & 63 & 314 & 106 & 4 \\
\hline KoRV flanks $>4$ bp & 6521 & 9200 & 392 & 2347 & 1158 & 28 \\
\hline Unique insertion sites after clustering & 66 & 182 & 126 & 538 & 862 & 24 \\
\hline Shared insertion sites after clustering & 15 & 28 & 17 & 134 & 25 & 0 \\
\hline Internal KoRV reads & 212 & 223 & 141 & 1406 & 151 & 14 \\
\hline Total target enrichment products identified & 22542 & 10950 & 1029 & 5559 & 1495 & 83 \\
\hline Total sequences after PCR duplicate removal* & 714929 & & 1188365 & & 11675245 & \\
\hline Efficiency of target enrichment(\%) & 4.68 & & 0.55 & & 0.01 & \\
\hline Number of hits to wallaby genome by blast & 1617 & & 136366 & & 1915781 & \\
\hline Estimated ratio of off-target enrichment(\%) & 0.23 & & 11.48 & & 16.41 & \\
\hline
\end{tabular}

* The total number of sequences after PCR duplicate removal equals the total number of sequences before pairwise alignment 


\section{Table 4(on next page)}

Table 4. Optimized parameters for clustering 
Table 4. Optimized parameters for clustering

Method

PEC

SPEX

Hybridization

Insertion site orientation

5 ' end

3 ' end

5 ' end

$1.00 \mathrm{E}-1.00 \mathrm{E}-$

1.00E-

E-value for all versus all blast

17

20

30

3' end

Capture

Inflation value for clustering

22

4

1.4

$1.00 \mathrm{E}$

5 ' end $\quad 3$ ' end

1.00E-

15

1.00E-15

1

46

16 